\title{
A CRETACEOUS PHONOLITE DYKE FROM THE TOMAHAWK RIVER, NORTHEAST TASMANIA
}

\author{
by J. L. Everard, F. L. Sutherland and H. Zwingmann \\ (with 11 tables, 13 text-figures and six plates)
}

\begin{abstract}
Everarm, J. L., Sutherland, F. L. \& Zwingmann, H., 2004 (29.x): A Cretaceous phonolite dyke from the Tomahawk River, northeastTasmania. Papers and Proceedings ofthe Royal Society of Tasmania 138: 11-33.

https://doi.org/10.26749/rstpp.138.11 ISSN 0080-4703.Tasmanian Geological Survey, Mineral Resources Tasmania, PO Box 56, Rosny Park, Tasmania 7018, Australia (JLE); Geodiversity Research Centre, Australian Museum, 6 College Street, Sydney, New South Wales 2010, Australia (FLS); CSIRO Division of Petroleum Resources and Centre of Excellence in Mass Spectrometry, School of Applied Geology, Curtin University, Western Australia 6102, Australia (HZ). ${ }^{*}$ Author for correspondence.

Field mapping and ground magnetics indicate that a phonolite dyke, which intrudes Devonian granite in the upper Tomahawk River area, Tasmania, is $3.2 \mathrm{~km}$ long and shallowly west-dipping wirh a true thickness of $10-15 \mathrm{~m}$. The rock contains microphenocrysts of anorthoclase, sparse fayalitic olivine and low-Ti magnetite, and rare biotite in a groundmass of aligned alkali feldspar laths, with interstitial clinopyroxene (hedenbergite to aegirine), amphibole (either hastingsite or arfvedsonite), sodalite, nepheline, analcime and accessory apatite. It is peralkaline and exceptionally strongly evolved, even for phonolites, with very low $\mathrm{TiO}_{2}, \mathrm{MgO}$ and $\mathrm{CaO}$, and high $\mathrm{Na}_{2} \mathrm{O}$ and incompatible elements. However, relatively high $\mathrm{Sr}$ and $\mathrm{Ba}$ and the absence of a negative Eu anomaly suggest little feldspar fractionation in its petrogenesis. Its Late Cretaceous age (K/Ar 80.4 $\pm 1.6 \mathrm{Ma},{ }^{40} \mathrm{Ar} /{ }^{34} \mathrm{Ar}$ plateau $75.8 \pm 0.3 \mathrm{Ma},{ }^{40} \mathrm{Ar} /{ }^{31)} \mathrm{Ar}$ total fusion $76 \pm 3.1 \mathrm{Ma}$ ) and geochemistry are unique for Tasmania and it represents a newly recognised, although very minor, igneous episode. It may be related to a change in dynamic regime following continental rifting, the opening of rhe Tasman Sea and the subsidence of Bass Basin.
\end{abstract}

Key Words: phonolite, peralkaline rocks, pyroxenes, amphiboles, arfvedsonite, $\mathrm{K} / \mathrm{Ar}$ dating, ${ }^{40} \mathrm{Ar} /{ }^{39} \mathrm{Ar}$ dating, Cretaceous, Tasmania.

\section{INTRODUCTION}

Phonolites are felsic, volcanic or hypabyssal igneous rocks which, despite their relatively high $\mathrm{SiO}_{2}$ content, are undersaturated in silica because of their very high alkali content $\left(\mathrm{Na}_{2} \mathrm{O}+\mathrm{K}_{2} \mathrm{O}\right)$. Their most voluminous development is in and around the East African Rift Valley (e.g., Lippard 1973, Price et al. 1985). Elsewhere they are relatively uncommon and usually subordinate to associated mafic rocks. There are well-documented Cainozoic examples in other continental settings, usually also related to rifting, such as the Rhine Graben (e.g., Wörner \& Schmincke 1984a, b), the Massif Central of France (Wilson et al. 1995) and the McMurdo Volcanic Province, which includes Mt Erebus, Antarctica (Kyle 1981, Kyle et al. 1992). Recently studied intra-plate oceanic examples occur on Tristan da Cunha (Le Roex et al. 1990), Tenerife (Ablay et al. 1998), Fernando de Noronha and Trinidade (Weaver 1990), Rarotonga (Thompson et al. 2001), Kaula, Hawaii (Garcia et al. 1986) and McDonald Island, Southern Ocean (Quilty \& Wheller 2000). These are associated with ocean island basalts (OIB) and, together with the McMurdo Volcanic Province, appear to be related to upwelling mantle plumes ("hotspots"). Phonolites are rare in subduction-related or orogenic environments (e.g., Keller 1983, Sorensen 1974).

There are limited occurrences of phonolite, of Jurassic to Late Cainozoic age, in eastern Australia (Ewart et al. 1985, Knutson 1989, Sutherland et al. 1996). They have not been recorded previously in Tasmania, although feldspathoidal syenite, their approximate plutonic equivalent, is a subordinate component of the Cretaceous Cygnet alkaline complex (Edwards 1947, Ford 1983, Taheri \& Bottrill 1999). A plug of Cainozoic basalt at Droughty Point near
Hobart contains small $(\leq 40 \mathrm{~mm})$ coarse-grained late-stage segregations, some of which approach mafic phonolite in composition (Sutherland 1976).

\section{PREVIOUS WORK}

Baillie (1972) reported outcrops of dark green "lamprophyre" at three localities (EQ641638, EQ638646, EQ636652 (grid references refer to $1: 25,000$ Monarch (5646) and 1:100,000 Cape Portland (8416) topographic sheets; datum is AGD66) in the valley of the Tomahawk River, about 9-12 km south of Tomahawk township. They were thought to indicate a NNW-trending post-granitic dyke of unknown length but not more than $6 \mathrm{~m}$ wide. The rock was described as consisting of phenocrysts $(25 \%)$ of oriented albite $(80 \%)$, olivine $(15 \%)$, lamprobolite amphibole $(4 \%)$ and pyrite $(1 \%)$ in an extremely fine-grained panidiomorphic granular groundmass containing a faintly green mineral, possibly actinolite.

On the Boobyalla 1:50,000 geological map (Baillie et al. 1978), the dyke is labelled "Ca?", a unit also used for Cretaceous appinitic rocks near Cape Portland (Jennings \& Sutherland 1969). The dyke is shown as extending for about $1.5 \mathrm{~km}$, partly concealed by Quaternary alluvium, with another small exposure $1.5 \mathrm{~km}$ further north, slightly offset to the east (EQ635664).

The local country rock is granite, comprising the Poimena and Little Mt Horror plutons of the Blue Tier Batholith (figs 1, 2; Groves 1977). Probably the most reliable published radiometric date from the Poimena pluton is a Rb-Sr mineral isochron of $379 \pm 3 \mathrm{Ma}$ (Middle Devonian) (McKenzie et al. 1988), although an unpublished SHRIMP U-Pb zircon age is about 5 Myr older (L. P. Black pers. comm.). On the 


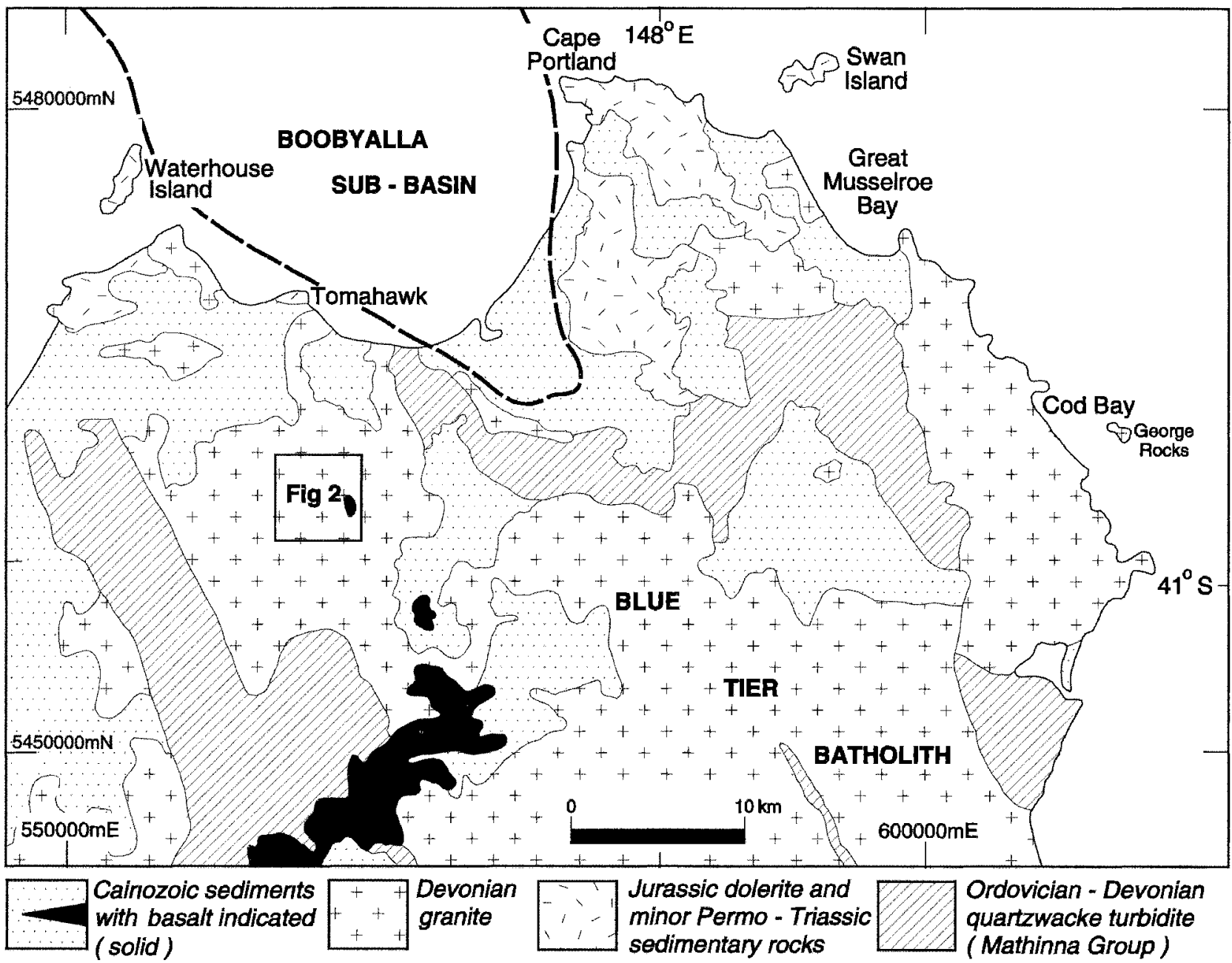

FIG. 1 - Simplified geology of part of northeast Tasmania showing location of Tomahawk River dyke and localities mentioned in text.

basis of intrusive relationships, the Little Mt Horror pluton is thought to be slightly younger than the Poimena pluton (Baillie 1972, McClenaghan et al. 1982).

\section{FIELD RELATIONS AND GEOPHYSICS}

The phonolite is a tough, dark bluish- to greenish-grey, fine-grained, massive aphanitic rock with locally well-developed platy jointing. Eight samples collected for petrological examination (table 1) are registered and stored at Mineral Resources Tasmania, Mornington.

The dyke can be traced along strike for about $3.2 \mathrm{~km}$ and has a slightly arcuate NNW to northerly trend (fig. 2). Its most southerly exposure is on the west bank of the Tomahawk River (EQ64106364), beyond which only granite was encountered. To the north, the dyke largely forms float on a series of low rocky knolls in rough pasture and light forest. In small cliffs just west of the river (EQ63376572), a steep western contact with fine-grained granite is exposed; here the dyke is about $20 \mathrm{~m}$ wide. About $400 \mathrm{~m}$ further north (near 63406620) a fault may offset the dyke dextrally by about $100 \mathrm{~m}$, but outcrop is poor and a change in strike is possible. The dyke re-appears immediately west of a small gorge in the river (EQ63516633), and scattered float and rubbly outcrop continue for a further $400 \mathrm{~m}$ to EQ63476672.
The phonolite has a moderately high mean magnetic susceptibility of about $5.35 \times 10^{-3}$ SI units (table 1) but it is not apparent on images of the area derived from regional aeromagnetic data (BMR, 1985). However, this survey had a $1.5 \mathrm{~km}$ line spacing and a $150-1000 \mathrm{~m}$ terrain clearance, probably inadequate to resolve any associated anomaly.

In order to constrain the dimensions and orientation of the body, six ground magnetometer traverses were measured across its southern segment, roughly perpendicular to strike (fig. 2). Survey details, data and interpretation are described in detail by Everard (2004).

The two northern profiles ( $A-A^{\prime}$ and $\left.B-B^{\prime}\right)$ each contain an asymmetric anomaly peaking at about $60 \mathrm{nT}$ above the local regional field, accompanied by a smaller negative anomaly to the east. They are easily modelled by a dipping dyke with a true thickness of $10-15 \mathrm{~m}$, dipping west at $18-31^{\circ}$ and extending very close to the surface. Profile $\mathrm{D}-\mathrm{D}^{\prime}$ is similar but was not modelled due to distortion of the field by several wire fences.

Profile C-C' defines a much broader and more subdued (15 nT) anomaly, consistent with a dyke dipping west at about $30^{\circ}$. It may have a maximum true thickness of about $13 \mathrm{~m}$ at a depth of $40 \mathrm{~m}$, tapering out both downward and within about $2 \mathrm{~m}$ of the surface.

Profile E-E' is complex with several distinct peaks of up to about $50 \mathrm{n} \mathrm{T}$ spread out over an east-west distance of about $120 \mathrm{~m}$, accompanied by a distinct negative anomaly to the 


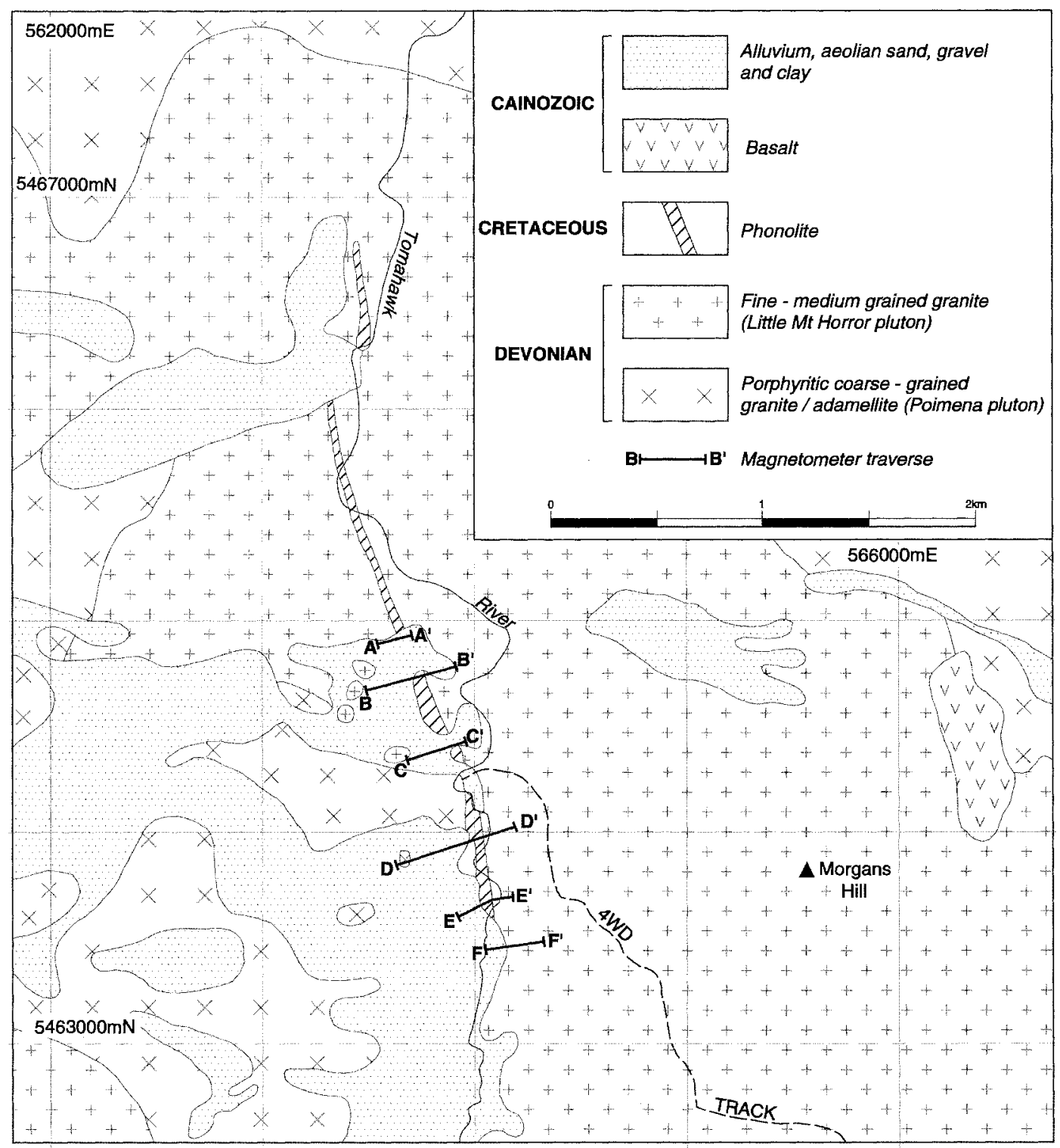

FIG. 2 - Detailed geology of upper Tomahawk River area (modified after Baillie et al. 1978) showing phonolite dyke and location of magnetometer traverses.

TABLE 1

Sample locations and physical properties

\begin{tabular}{|c|c|c|c|c|c|c|c|c|}
\hline \multirow[t]{2}{*}{ Field no. } & \multirow[t]{2}{*}{ Reg. no. } & metres $\mathrm{E}$ & metres $\mathrm{N}$ & \multicolumn{4}{|c|}{ Magnetic susceptibility $\left(\times 10^{-3} \mathrm{SI}\right)$} & \multirow{2}{*}{$\begin{array}{c}\text { Density } \\
\mathrm{t}^{\mathrm{m}} \mathrm{m}^{3}\end{array}$} \\
\hline & & \multicolumn{2}{|c|}{ datum:AGD66 } & $\mathrm{N}$ & mean & $\min$ & $\max$ & \\
\hline $\mathrm{PHN}^{\mathrm{a}}$ & C108641 & 563640 & 5465010 & & & & & \\
\hline $\mathrm{PHS}^{\mathrm{a}, \mathrm{b}}$ & $\mathrm{C} 108642$ & 563770 & 5464660 & & & & & \\
\hline $\mathrm{CPJ} 32^{\mathrm{a}, \mathrm{b}}$ & R006569 & 564100 & 5463640 & 10 & 5.64 & 4.85 & 6.53 & 2.642 \\
\hline $\mathrm{CPJ} 33^{a}$ & R006570 & 564110 & 5463690 & & & & & \\
\hline CPJ38 & $\mathrm{R} 004373$ & 563770 & 5464630 & & & & & \\
\hline CPJ39 & R004374 & 563510 & 5465380 & & & & & \\
\hline CPJ40 & R004375 & 563510 & 5466440 & 10 & 6.19 & 5.22 & 7.44 & 2.628 \\
\hline $\mathrm{CPJ} 41^{\mathrm{a}}$ & R004376 & 563350 & 5465990 & 20 & 4.78 & 2.45 & 6.31 & 2.605 \\
\hline
\end{tabular}

a mineral (electron microprobe) analyses (tables 4-10)

${ }^{b}$ whole rock analyses (table 11) 
west. Modelling suggests that here the main dyke may be split into five sub-parallel splays, none more than about 10 $m$ thick, each dipping west at about $30^{\circ}$. These may taper and pinch out southward, as profile F-F', about $200 \mathrm{~m}$ south of the southernmost phonolite outcrop, contains two small overlapping positive anomalies of about $12 \mathrm{nT}$ and 8 $\mathrm{nT}$, consistent with two thin $(3-4 \mathrm{~m})$ west-dipping dykes concealed by about $10 \mathrm{~m}$ of overburden.

\section{GEOCHRONOLOGY}

\section{K-Ar Analysis}

The selected sample CPJ 32, with least alteration of K-bearing phases, was ground to $750 \mu \mathrm{m}$. K contents were determined by atomic absorption (Varian AA20), following procedures after Heinrichs \& Herrmann (1990). The pooled error of duplicate $\mathrm{K}$ determinations and standards is better than $2 \%$. $\mathrm{K}-\mathrm{Ar}$ isotope determinations followed Bonhomme et al. (1975) and spiked isotopes were measured in a high sensitivity VG 3600 noble-gas mass spectrometer. The ${ }^{38} \mathrm{Ar}$ spike was calibrated against biotite GA1550 (95.5 Ma, McDougall \& Roksandic 1974) together with measurements of standards (LP-6, HD-B1, GLO and GA 1550) and mass discrimination factors (airshots). Errors for Ar analysis were $<1 \%$ and ${ }^{40} \mathrm{Ar} /{ }^{36} \mathrm{Ar}$ values for airshots were $293.39 \pm 0.29(2 \sigma)$. The result with $2 \sigma$ error (table 2 ) used ${ }^{40} \mathrm{~K}$ abundances and decay constants recommended by Steiger \& Jäger (1977).

\section{${ }^{40} \mathrm{Ar}-{ }^{39} \mathrm{Ar}$ analysis}

A detailed account of the ${ }^{40} \mathrm{Ar}-{ }^{39} \mathrm{Ar}$ dating technique (McDougall \& Harrison 1999) underpins the dating presented here. A concentrate of separated feldspar from the rock was cleaned before wrapping in aluminium foil for loading into an aluminium package, along with biotite standard $\mathrm{HD}-\mathrm{B} 1$ (24.21 $\pm 0.32 \mathrm{Ma}$, Hess \& Lippolt 1994) to monitor the neutron flux gradient. The package was Cd-shielded and fast neutron irradiated in the McMaster University Nuclear Reactor, Hamilton, Canada, for 28.4 hours.

For infra-red laser step-heating, the mineral separate was loaded onto an aluminium palette and baked within an ultra-high vacuum laser sample chamber to $120^{\circ} \mathrm{C}$ overnight to remove absorbed atmospheric argon. A $110 \mathrm{~W}$ Spectron Laser systems continuous wave $\mathrm{Nd}-\mathrm{Y}$-Al-garnet $(=\lambda 1064$ $\mathrm{nm}$ ) laser was used to laser step treat the sample.

Gas cleanup and mass spectrometry removed active gases using $\mathrm{Zr}$-Al getter pumps and the remaining noble gases were equilibrated into a Mass Analyser Product 215-50 static mass spectrometer with a Balzers multiplier detector. The Ar sensitivity of the ms multiplier detector was $2.71 \mathrm{x}$ $10^{-10} \mathrm{~cm}^{3} /$ Volt. A Lab View program was used to measure isotope peak masses from 35-41 (10 times) in sequence lasting -15 minutes, via a peak hopping routine. Blank analyses were run before and after.

Age calculation involved extrapolating peak intensities back to the inlet time and correcting for extraction line blanks, ms backgrounds, $\mathrm{ms}$ discrimination $\left({ }^{40} \mathrm{Ar} /{ }^{36} \mathrm{Ar}\right.$ ratio of 281.0) and reactor interferences. The $\mathrm{Ca}$ and $\mathrm{K}$ correction factors in the data reduction were ${ }^{39} \mathrm{Ar}^{37} \mathrm{Ar}_{\mathrm{Ca}}=$ $0.00065,{ }^{36} \mathrm{Ar}^{37} \mathrm{Ar}_{\mathrm{Ca}}=0.000255$ and $\left.{ }^{40} \mathrm{Ar}^{36} \mathrm{Ar}_{\mathrm{K}}=0.0015\right)$. Errors quoted are $1 \sigma$ and $\mathrm{Ar}$-Ar ages were calculated using decay constants of Steiger \& Jäger (1977). Weighred mean

\begin{tabular}{lccccc}
\multicolumn{6}{c}{ TABLE 2 } \\
K-Ar analysis \\
\hline Sample & $\begin{array}{c}\% \mathrm{~K} \\
(\text { dupli- } \\
\text { cate })\end{array}$ & $\begin{array}{c}{ }^{40} \mathrm{Ar}^{\mathrm{a}} \\
\left(\times 10^{-3} \mathrm{~mol} / \mathrm{g}\right)\end{array}$ & ${ }^{40} \mathrm{Ar}^{40} \mathrm{Ar}_{\text {total }}$ & $\begin{array}{c}\text { Age } \\
(\mathrm{Ma})\end{array}$ & $\begin{array}{c}\text { Error } \\
( \pm 2 \delta)\end{array}$ \\
\hline CPJ32 & 3.31 & 4.720 & 0.927 & 80.40 & 1.60 \\
\hline $\begin{array}{l}{ }^{2} \text { Denotes radiogenic argon. } \\
\text { Constants: }\end{array}{ }^{40} \mathrm{~K}=0.01167$ atom $96 ; \lambda \beta=4.962, \lambda \varepsilon=0.581 \mathrm{x}$ \\
$10^{-10} \mathrm{y}^{-1}$
\end{tabular}

ages were calculated ( $2 \sigma$ errors) using the Ludwig (2001) Isoplot program (fig. 3).

\section{Results}

The K-Ar results (table 2) give an age of $80.40 \pm 1.60 \mathrm{Ma}$. The Ar-Ar analysis (table 3, fig. 3) gives a weighted mean age of $75.77 \pm 0.32 \mathrm{Ma}$ from steps $4-5$ (lowest-Ca steps and highest ${ }^{40} \mathrm{Ar}{ }^{*}$ yields) and a total fusion age of $76 \pm 3.1 \mathrm{Ma}$. There was evidence of mixing with a $\mathrm{Ca}$-rich phase in the feldspar separate. The total fusion $\mathrm{Ar}-\mathrm{Ar}$ and $\mathrm{K}-\mathrm{Ar}$ age are within error and with the weighted mean $\mathrm{Ar}-\mathrm{Ar}$ age confirm a Late Cretaceous age for the phonolite crystallisation, with age limits between 75 and $81 \mathrm{Ma}$. On recent time-scales (e.g., Young \& Laurie 1996) this is within the Campanian (73-83 Ma).

\section{PETROGRAPHY}

In thin section (pls 1-6), the samples are similar. Most are slightly porphyritic (pl. 1) to seriate (pl. 2), with microphenocrysts of anorthoclase $(\leq 1.5 \mathrm{~mm}$ but commonly 500 $\mu \mathrm{m}-1 \mathrm{~mm}$ long) grading down in grain size to a usually pilotaxitic groundmass. Sparse to rare, usually corroded microphenocrysts include fayalite $(\leq 0.1-1.0 \%$ by volume), magnetite (c. $0.3-1 \%$ ) and rare biotite. These minerals are rare in the groundmass, probably due to a reaction relationship with it (pls 3, 4, 5).

The groundmass (pls 5,6) consists of flow-aligned laths (commonly 50-100 $\mathrm{\mu m}$ long) of alkali feldspar (mainly anorthoclase), small platelets and prisms of clinopyroxene (hedenbergite to aegirine) and amphibole (hastingsite and/or arfvedsonite), together with interstitial sanidine, feldpathoids, zeolites, minor pale brown, slightly turbid glass, accessory apatite, and sometimes traces of biotite.

Electron microprobe analyses (see below) and Xray diffraction of powdered samples indicate that the feldspathoids are nepheline and sodalite. The main zeolite is analcime, with substantial natrolite in CPJ39. Their identification is supported by the disappearance of their diffraction peaks after treatment of the powders with dilute $\mathrm{HCl}$, which dissolves these minerals (R. N. Woolley, pers. comm.). Reconnaissance scanning electron microscope work (R. S. Bottrill) suggests the presence of small amounts of a $\mathrm{Na}-\mathrm{Ca}$ silicate, possibly pectolite, as minute grains in one sample (PHS). The presence of pyrite, reported in the dyke by Baillie (1972), has not been substantiated.

There are small variations between samples in the abundance of feldspar microphenocrysts, groundmass 


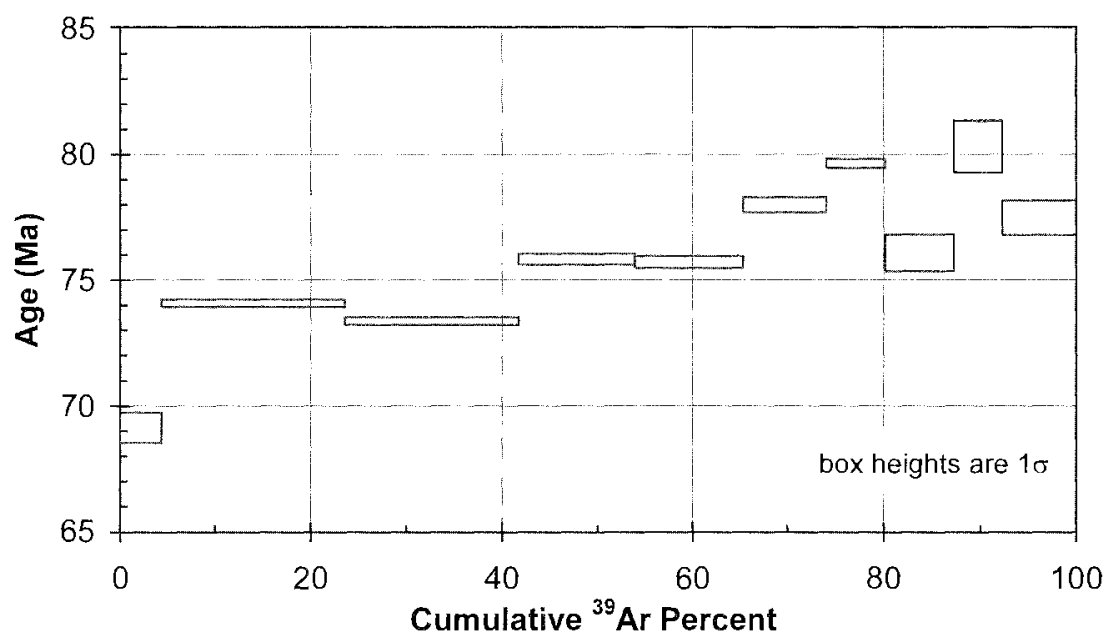

FIG. 3 - Step heating age spectrum (apparent age versus cumulative ${ }^{39}$ Ar released) for ${ }^{40} \mathrm{Ar}{ }^{39} \mathrm{Ar}$ analysis, Tomahawk phonolite sample CPJ32.

TABLE 3

${ }^{40} \mathrm{Ar}^{39}{ }^{39}$ Ar analysis, Tomahawk River phonolite; sample 412 (K-feldspar from sample CPJ32)

\begin{tabular}{lccccccccc}
\hline Step & Age $(\mathrm{Ma})$ & ${ }^{40} \mathrm{Ar}{ }^{* 39} \mathrm{Ar}$ & ${ }^{40} \mathrm{Ar}{ }^{39} \mathrm{Ar}$ & ${ }^{38} \mathrm{Ar} r^{39} \mathrm{Ar}$ & ${ }^{37} \mathrm{Ar}{ }^{39} \mathrm{Ar}$ & ${ }^{36} \mathrm{Ar} l^{39} \mathrm{Ar}$ & ${ }^{39} \mathrm{Ar}\left(10^{-12} \mathrm{~cm}^{3}\right)$ & $40 \mathrm{Ar} r^{*}(\%)$ \\
\hline 1 & $69.13 \pm 0.69$ & $5.90 \pm 0.05$ & $7.49 \pm 0.01$ & $0.01287 \pm 0.00035$ & $0.06238 \pm 0.00417$ & $0.00539 \pm 0.00017$ & $77.74 \pm 0.06$ & 78.7 \\
2 & $74.08 \pm 0.39$ & $6.33 \pm 0.01$ & $7.08 \pm 0.01$ & $0.01299 \pm 0.00011$ & $0.04164 \pm 0.00095$ & $0.00539 \pm 0.00004$ & $342.45 \pm 0.22$ & 89.3 \\
3 & $73.36 \pm 0.39$ & $6.26 \pm 0.01$ & $6.79 \pm 0.01$ & $0.01532 \pm 0.00008$ & $0.03488 \pm 0.00100$ & $0.00178 \pm 0.00004$ & $324.82 \pm 0.24$ & 92.2 \\
4 & $75.83 \pm 0.43$ & $6.48 \pm 0.02$ & $6.97 \pm 0.01$ & $0.01363 \pm 0.00012$ & $0.02630 \pm 0.00067$ & $0.00167 \pm 0.00006$ & $218.22 \pm 0.16$ & 92.9 \\
5 & $75.72 \pm 0.44$ & $6.47 \pm 0.02$ & $6.88 \pm 0.01$ & $0.01335 \pm 0.00013$ & $0.02598 \pm 0.00143$ & $0.00139 \pm 0.00007$ & $204.05 \pm 0.16$ & 94.0 \\
6 & $78.00 \pm 0.48$ & $6.67 \pm 0.03$ & $7.26 \pm 0.01$ & $0.01390 \pm 0.00017$ & $0.02833 \pm 0.00186$ & $0.00199 \pm 0.00009$ & $156.00 \pm 0.09$ & 91.9 \\
7 & $79.64 \pm 0.43$ & $6.81 \pm 0.02$ & $7.33 \pm 0.02$ & $0.01392 \pm 0.00013$ & $0.02709 \pm 0.00006$ & $0.00174 \pm 0.00000$ & $108.85 \pm 0.24$ & 93.0 \\
8 & $76.08 \pm 0.82$ & $6.50 \pm 0.06$ & $7.38 \pm 0.01$ & $0.01415 \pm 0.00024$ & $0.05120 \pm 0.00230$ & $0.00298 \pm 0.00021$ & $126.75 \pm 0.08$ & 88.1 \\
9 & $80.31 \pm 1.09$ & $6.87 \pm 0.09$ & $7.49 \pm 0.01$ & $0.01360 \pm 0.00030$ & $0.04923 \pm 0.00972$ & $0.00210 \pm 0.00030$ & $89.96 \pm 0.08$ & 91.7 \\
10 & $77.47 \pm 0.78$ & $6.62 \pm 0.06$ & $7.44 \pm 0.02$ & $0.01256 \pm 0.00020$ & $0.03444 \pm 0.00672$ & $0.00276 \pm 0.00020$ & $137.22 \pm 0.27$ & 89.0 \\
\hline
\end{tabular}

Total fusion age $=75.96 \pm 3.10 \mathrm{Ma}$

Irradiation standard used $=\mathrm{HD}-\mathrm{B} 1$ biotite $(24.21 \pm 0.32 \mathrm{Ma})$

$\mathrm{J}$ value $=0.006510 \pm 0.000032$

$1 \sigma$ errors

Weighted mean age (steps $4-5)=75.77 \pm 0.60 \mathrm{Ma}(2 \sigma)$

grain-size, development of flow lamination and degree of alteration (especially of feldspar). In particular, CPJ38 and CPJ39 (pl. 3) are aphyric and relatively coarse-grained with randomly oriented feldspars (typically 400-600 $\mu \mathrm{m}$ x 50-100 $\mu \mathrm{m}$ ), and may be slower-cooled rocks from near the centre of the dyke.

\section{MINERALOGY AND MINERAL CHEMISTRY}

Minerals from five polished thin sections were analysed using the Cameca SX-50 electron microprobe at the University of Tasmania. Representative analyses and calculated cation formulae are given in tables 4-10 and full data by Everard (2004).

The amphibole arfvedsonite is a new mineral species for Tasmania, although there is a very old and spurious record from Swan Island (Anon 1970).

\section{Olivine (fayalite)}

Pale yellow fayalite occurs sometimes as equant to oblong euhedral microphenocrysts ( $\leq 1 \mathrm{~mm}$ ) (pls 1,2) with slightly corroded, ragged margins, but commonly as deeply-embayed subhedra (typically 400-500 $\mu \mathrm{m}$ ) (pls 2, 4) or anhedral granules $(\mathrm{c} .100 \mu \mathrm{m})$ (pl. 3). Some of the larger microphenocrysts contain inclusions of apatite, as rod-like laths (up to $100 \mathrm{x}$ $15 \mu \mathrm{m}$ ) and hexagonal sections (up to $120 \mu \mathrm{m}$ across). In some samples, fayalite is altered to orange to amber-coloured fibrous alteration products (CPJ33) or dark red-brown "iddingsite" (PHS).

Analyses (table 4, fig. 4) are near-stoichiometric with significant $\mathrm{MnO}(\leq 4.25 \%)$ and $\mathrm{MgO}(\leq 3.83 \%)$ but a narrow compositional range $\left(\begin{array}{llll}\mathrm{Fa} & { }_{86.3-89.0} & \mathrm{Fo}_{4.4-9.2} & \mathrm{Jo}_{3.6-6.0}\end{array}\right)$. The $\mathrm{CaO}$ contents $(0.28-0.59 \%)$ are those of low pressure, volcanic olivine $(\geq 0.10 \%)$ rather than plutonic olivine $(\leq 0.10 \%)($ Simkin \& Smith 1970). 


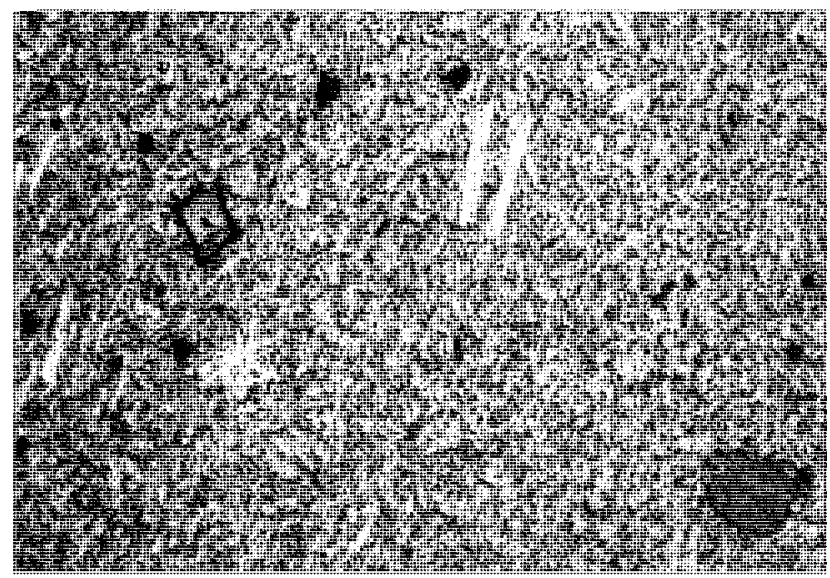

PLATE

Photomicrograph of phonolite (PHS), showing porphyritic texture with microphenocrysts of fayalite (left), anorthoclase (e.g., upper centre) and corroded biotite (lower right) in a fine-grained
pilotaxitic groundmass (plane polarised light, field of view 4.5 pilotaxitic gro
$\times 3.4 \mathrm{~mm}$ ).

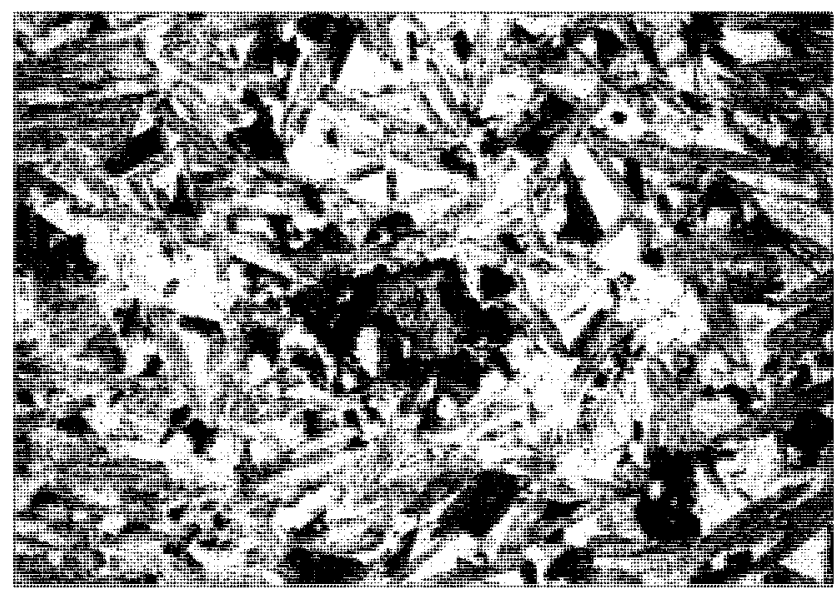

PLATE 3 Photomicrograph of phonolite (CPJ39), showing aphyric, relatively coarse-grained texture of mainly pyroxene and amphibole (dark),
turbid alkali feldspar (grey) and interstitial feldspathoids and zeolites (clear). A central corroded fayalite grain has a narrow reaction rim of biotite and magnetite (plane polarised light, field of view $4.5 \times 3.4 \mathrm{~mm}$ ).

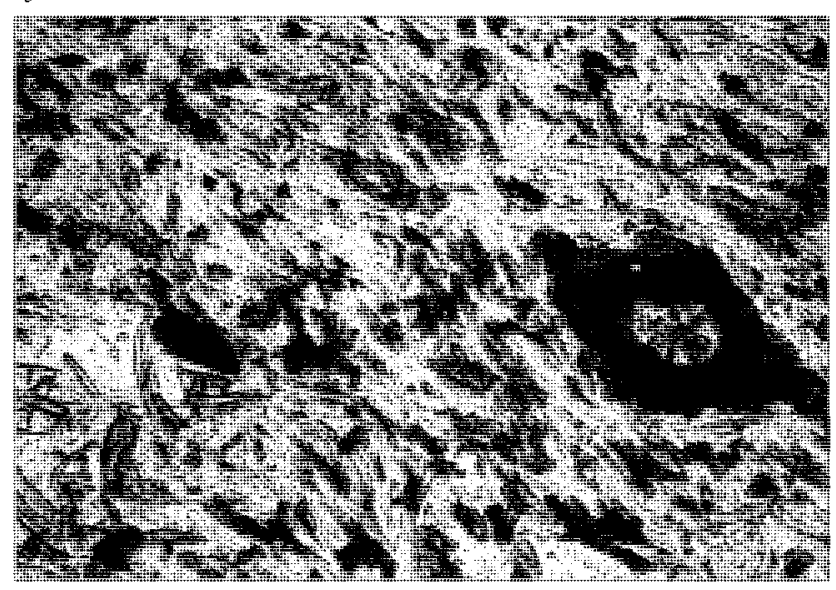

\section{PLATE 5}

Photomicrograph of phonolite (PHS) groundmass, showing Photomicrograph of phonolite (PHS) groundmass, showing prisms aligned alkali feldspar laths with prisms of aegirine (grey) and and sections of arfuedsonite (dark, e.g., left) and aegirine (e.g., arfuedsonite (dark grey, e.g., centre left). A fayalite granule (centre centre right) with feldspars, feldspathoids, zeolite and acicular right) has a reaction rim of arfvedsonite (plane polarised light, apatite (e.g., upper left)(plane polarised light, field of view 280 field of view $730 \times 550 \mu \mathrm{m}$ ).

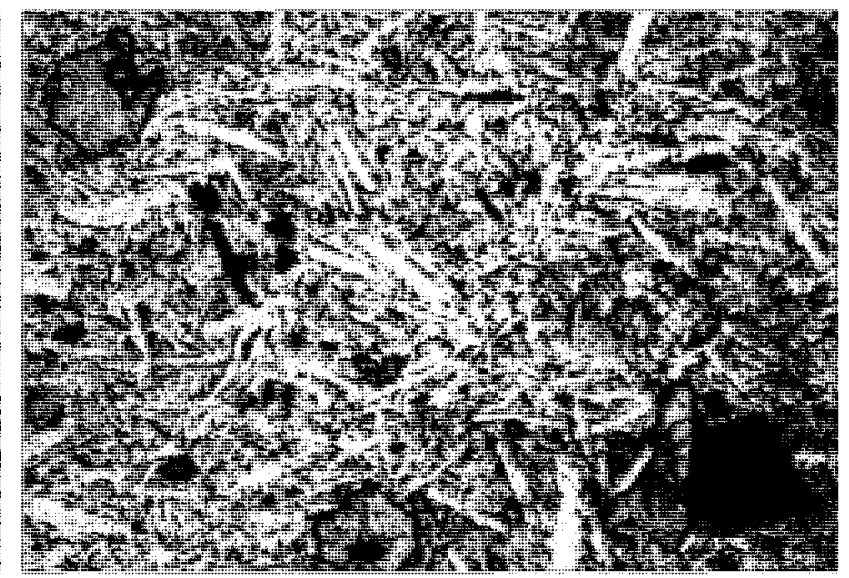

PLATE 2

Photomicrograph of phonolite (CPJ32), showing porphyritic to seriate texture with microphenocrysts of fayalite (upper left, centre bottom, lower right), magnetite (lower right) and anorthoclase
grading to the groundmass (plane polarised light, field of view $4.5 \times 3.4 \mathrm{~mm}$ ).

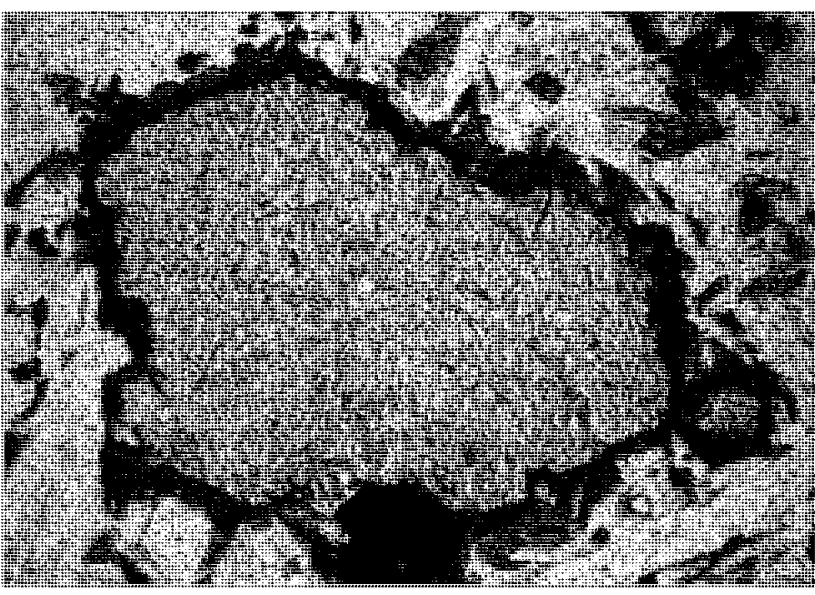

PLATE 4

Photomicrograph of phonolite (CPJ32) showing a corroded fayalite light, field of view $730 \times 550 \mu \mathrm{m}$ ).

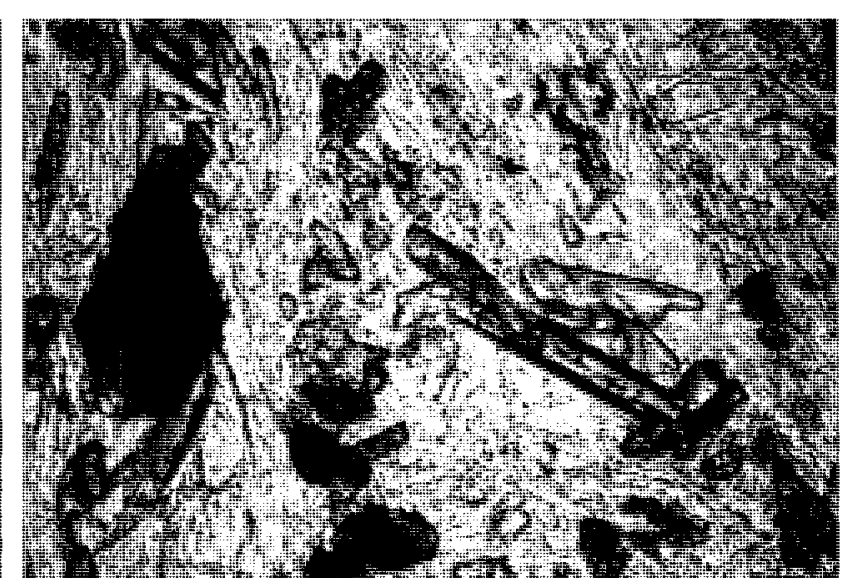

\section{PLATE 6} microphenocryst and abutting small magnetite microphenocryst 


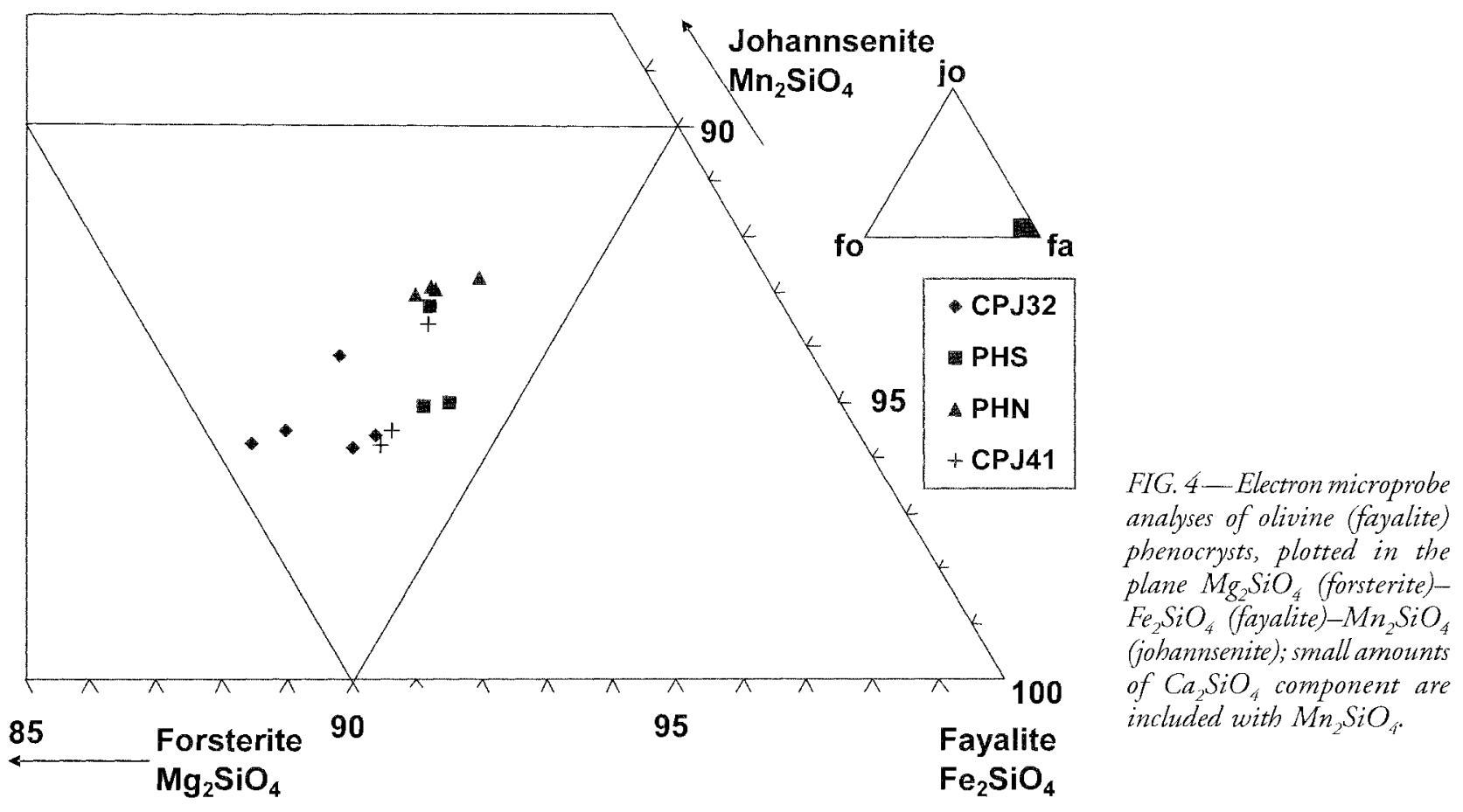

TABLE 4

Representative mineral analyses: olivine (fayalite) phenocrysts

\begin{tabular}{|c|c|c|c|}
\hline Sample & CPJ32 & PHS & PHN \\
\hline Analysis & $\mathrm{R} 1 / 1$ & $\mathrm{R} 7 / 4$ & $\mathrm{R} 5 / 1$ \\
\hline $\mathrm{SiO}_{2}$ & 30.40 & 29.87 & 29.52 \\
\hline $\mathrm{TiO}_{2}$ & - & 0.03 & 0.01 \\
\hline $\mathrm{Cr}_{2} \mathrm{O}_{3}$ & 0.02 & 0.01 & - \\
\hline $\mathrm{FeO}$ & 63.34 & 64.16 & 63.20 \\
\hline $\mathrm{MnO}$ & 2.75 & 3.21 & 4.18 \\
\hline $\mathrm{NiO}$ & 0.07 & - & - \\
\hline $\mathrm{MgO}$ & 3.83 & 2.42 & 1.78 \\
\hline $\mathrm{CaO}$ & 0.30 & 0.31 & 0.76 \\
\hline Total & 100.72 & 100.02 & 99.45 \\
\hline \multicolumn{4}{|c|}{ Cations calculated on the basis of $(O)=4$} \\
\hline $\mathrm{Si}$ & 0.995 & 0.995 & 0.993 \\
\hline Ti & - & 0.001 & - \\
\hline $\mathrm{Cr}$ & 0.001 & - & - \\
\hline $\mathrm{Fe}^{1 \mathrm{II}}$ & 1.734 & 1.787 & 1.778 \\
\hline $\mathrm{Mn}$ & 0.076 & 0.091 & 0.119 \\
\hline $\mathrm{Ni}$ & 0.002 & - & - \\
\hline $\mathrm{Mg}$ & 0.187 & 0.120 & 0.089 \\
\hline $\mathrm{Ca}$ & 0.011 & 0.011 & 0.028 \\
\hline Total & 3.005 & 3.004 & 3.007 \\
\hline $\mathrm{Mg \#}$ & 9.73 & 6.31 & 4.77 \\
\hline
\end{tabular}

\section{Magnetite}

Opaque microphenocrysts (pls 2, 4) are equant, angular, polygonal (squarish to pseudohexagonal) to irregular or skeletal grains, exceptionally up to $1.5 \times 1 \mathrm{~mm}$, but commonly $150-400 \mu \mathrm{m}$ across. They are identified in reflected light as magnetite, and can be drawn off the powdered samples by a magnet. Some of the larger microphenocrysts contain small $(\leq 50 \mu \mathrm{m})$ inclusions of biotite and euhedral apatite. Usually, opaque phases are absent in the groundmass, except for rare small (c. $50 \mu \mathrm{m})$ partly oxidised magnetite grains in PHN.

Analyses (table 5) match low-Ti magnetite $\left(\mathrm{TiO}_{2} \leq 6.24 \%\right)$ in which $\mathrm{Al}_{2} \mathrm{O}_{3}(\leq 3.29 \%)$ and $\mathrm{MnO}(\leq 0.60 \%)$ are minor components. These values correspond to $\leq 18.0$ mole \% ulvospinel and $\leq 4.6$ mole \% hercynite in solid solution.

\section{Biotite}

Biotite is an uncommon mineral in the phonolite, but has three distinct parageneses. All are iron-rich biotites (table 6) with low fluorine $(\leq 0.15 \%)$ and chlorine $(\leq 0.13 \%)$, with compositions between annite and siderophyllite (fig. 5a).

Biotite microphenocrysts ( $\alpha, \beta$ very dark sepia-brown, $\gamma$ pale honey-yellow $)$ are rare $(<0.1 \%)$, rounded and corroded, apparently partly resorbed anhedra, up to $700 \mathrm{x}$ $380 \mu \mathrm{m}$ across (pl. 1). They are iron-rich (Mg\# 8.4-18.7) with high tetrahedrally coordinated $\mathrm{Al}^{\text {iv }}$ (2.44-2.59 cations per formula unit), balanced by substitution of small amounts of $\mathrm{Ti}\left(\mathrm{TiO}_{2} \leq 1.54 \%\right)$ and $\mathrm{Al}^{\mathrm{vi}}$ into the octahedral sites. $\mathrm{MnO}(\leq 0.54 \%)$ and $\mathrm{CaO}(\leq 0.28 \%)$ are low.

Rare small ragged biotite is present in the groundmass of some samples. Analyses (from PHN) have similar Mg\# (9.0-13.4) and $\mathrm{Al}^{\mathrm{iv}}(2.35-2.50$ cations/formula unit) to the microphenocrysts but lower $\mathrm{TiO}_{2}(0.53-0.77 \%)$ (fig. 5b) and slightly higher $\mathrm{MnO}(0.60-1.62 \%)$ and $\mathrm{CaO}(\leq 0.57 \%)$. 
TABLE 5

Representative mineral analyses: magnetite

\begin{tabular}{|c|c|c|c|c|}
\hline Sample & CPJ32 & PHS & $\mathrm{CPJ} 33$ & CPJ41 \\
\hline Analysis & $\mathrm{R} 1 / 3$ & $\mathrm{R} 14 \mathrm{a} / \mathrm{l}$ & $\mathrm{m} 4$ & $\mathrm{R} 11 / 2$ \\
\hline $\mathrm{SiO}_{2}$ & 0.33 & 0.30 & 0.33 & 0.30 \\
\hline $\mathrm{TiO}_{2}$ & 4.72 & 2.24 & 6.24 & 1.81 \\
\hline $\mathrm{ZrO}_{2}$ & - & 0.08 & - & 0.06 \\
\hline $\mathrm{Al}_{2} \mathrm{O}_{3}$ & 3.17 & 1.29 & 3.00 & 1.87 \\
\hline $\mathrm{Cr}_{2} \mathrm{O}_{3}$ & 0.02 & 0.03 & - & 0.01 \\
\hline $\mathrm{FeO}_{\mathrm{r}}$ & 84.98 & 89.20 & 82.43 & 90.04 \\
\hline $\mathrm{MnO}$ & 0.44 & 0.59 & 0.66 & 0.42 \\
\hline $\mathrm{ZnO}$ & 0.02 & - & 0.25 & - \\
\hline $\mathrm{MgO}$ & 0.19 & 0.05 & 0.15 & 0.03 \\
\hline $\mathrm{CaO}$ & 0.01 & - & 0.02 & 0.01 \\
\hline Total & 93.89 & 93.78 & 93.09 & 94.54 \\
\hline \multicolumn{5}{|c|}{ cations calculated on the basis of $Z=3,(O)=4$} \\
\hline Ti & 0.135 & 0.064 & 0.180 & 0.052 \\
\hline $\mathrm{Zr}$ & - & 0.002 & - & 0.001 \\
\hline $\mathrm{Al}$ & 0.142 & 0.058 & 0.136 & 0.083 \\
\hline $\mathrm{Cr}$ & 0.001 & 0.001 & - & - \\
\hline $\mathrm{Fe}^{\mathrm{III}}$ & 1.588 & 1.809 & 1.504 & 1.811 \\
\hline $\mathrm{Fe}^{\mathrm{II}}$ & 1.109 & 1.044 & 1.142 & 1.037 \\
\hline $\mathrm{Mn}$ & 0.014 & 0.019 & 0.022 & 0.013 \\
\hline $\mathrm{Zn}$ & 0.001 & - & 0.007 & - \\
\hline $\mathrm{Mg}$ & 0.011 & 0.003 & 0.009 & 0.002 \\
\hline $\mathrm{Ca}$ & 0.001 & - & 0.001 & - \\
\hline \multicolumn{5}{|c|}{ end-members (mole \%) } \\
\hline $\mathrm{Fe}_{2}^{\mathrm{II}} \mathrm{TiO}_{4}$ & 13.47 & 6.59 & 18.00 & 5.26 \\
\hline $\mathrm{Fe}^{\mathrm{iT}} \mathrm{Al}_{2} \mathrm{O}_{4}$ & 4.47 & 0.68 & 2.98 & 2.63 \\
\hline $\mathrm{Fe}^{\mathrm{II}} \mathrm{Fe}_{2}^{\mathrm{III}} \mathrm{O}_{4}$ & 79.41 & 90.46 & 75.21 & 90.56 \\
\hline other & 2.65 & 2.27 & 3.81 & 1.55 \\
\hline
\end{tabular}

Fayalite and magnetite microphenocrysts commonly exhibit a narrow $(5-10 \mu \mathrm{m})$ discontinuous reaction rim of granular biotite (pleochroic from deep orange-brown to almost opaque), surrounded by a concentration of green amphibole and pyroxene which passes into the groundmass (pls 3, 4). The biotite rims are low in $\mathrm{TiO}_{2}(\leq 0.9 \%)$, relatively high in $\mathrm{MnO}(0.9-1.69 \%)$ and similar to groundmass biotite. A biotite inclusion within a magnetite phenocryst in CPJ33 has exceptionally low $\mathrm{Mg} \#(0.70)$ and relatively high $\mathrm{TiO}_{2}(1.81 \%)$.

Resorption of the biotite microphenocrysts suggests disequilibrium with the melt at emplacement. However, they are probably not xenocrysts derived from the granitic country rock, as the phonolite lacks other granite-derived xenocrysts such as quartz or plagioclase. Furthermore, biotites from the Poimena and Little Mount Horror plutons have typically higher $\mathrm{Mg} \#$ and $\mathrm{TiO}_{2}(2.88-5.55 \%$ )(Groves 1977, McClenaghan \& Williams 1982). Elsewhere in eastern Tasmania, some alkali feldspar granites contain very iron-rich, low $\mathrm{Ti}$ biotites, but these have generally lower tetrahedral $\mathrm{Al}$ and contain much more $\mathrm{F}(0.6-4.2 \%)$ (Groves 1977, J. L. Everard unpublished data).
TABLE 6

Representative mineral analyses: biotite

\begin{tabular}{|c|c|c|c|c|c|c|}
\hline Sample & CPJ32 & PHS & CPJ32 & CPJ32 & CPJ33 & PHN \\
\hline Analysis & $\mathrm{R} 2$ & $\mathrm{R} 3 / 1$ & $\mathrm{R} 3 / 3$ & $\mathrm{R} 6 / 9$ & $\mathrm{R} 1 / 2$ & $\mathrm{R} 5 / 3$ \\
\hline Type & pheno & pheno & $\begin{array}{c}\text { rim on } \\
\mathrm{mt}\end{array}$ & $\begin{array}{c}\operatorname{rim} \text { on } \\
\mathrm{fa}\end{array}$ & $\begin{array}{c}\text { incl in } \\
\mathrm{mt}\end{array}$ & gmass \\
\hline $\mathrm{SiO}_{2}$ & 33.24 & 32.70 & 33.01 & 33.01 & 32.82 & 32.31 \\
\hline $\mathrm{TiO}_{2}$ & 1.66 & 1.30 & 0.69 & 0.35 & 1.81 & 0.65 \\
\hline $\mathrm{ZrO}_{2}$ & na & na & 0.02 & 0.08 & na & 0.03 \\
\hline $\mathrm{Al}_{2} \mathrm{O}_{3}$ & 14.44 & 13.98 & 11.79 & 10.32 & 10.13 & 11.45 \\
\hline $\mathrm{Cr}_{2} \mathrm{O}_{3}$ & - & 0.02 & na & na & - & 0.02 \\
\hline $\mathrm{V}_{2} \mathrm{O}_{3}$ & 0.02 & - & na & na & - & na \\
\hline $\mathrm{FeO}_{\mathrm{t}}$ & 33.08 & 36.68 & 38.84 & 38.19 & 40.83 & 36.99 \\
\hline $\mathrm{MnO}$ & 0.30 & 0.51 & 0.69 & 1.01 & 0.54 & 0.60 \\
\hline $\mathrm{ZnO}$ & 0.12 & 0.14 & na & na & 0.02 & na \\
\hline $\mathrm{NiO}$ & 0.07 & - & na & na & na & - \\
\hline $\mathrm{MgO}$ & 4.12 & 1.89 & 1.10 & 1.85 & 0.16 & 2.06 \\
\hline $\mathrm{CaO}$ & - & 0.02 & 0.04 & 0.14 & 0.07 & 0.08 \\
\hline $\mathrm{Na}_{2} \mathrm{O}$ & 0.70 & 0.61 & 0.75 & 0.54 & 0.24 & 0.43 \\
\hline $\mathrm{K}_{2} \mathrm{O}$ & 8.46 & 7.67 & 7.95 & 7.71 & 7.75 & 7.67 \\
\hline $\mathrm{SrO}$ & - & 0.08 & na & na & - & na \\
\hline $\mathrm{BaO}$ & 0.65 & 0.47 & na & na & - & na \\
\hline $\mathrm{F}$ & 0.08 & 0.09 & 0.01 & 0.08 & 0.14 & 0.15 \\
\hline $\mathrm{Cl}$ & 0.13 & 0.11 & 0.04 & 0.04 & 0.01 & 0.08 \\
\hline $\mathrm{O}=(\mathrm{F}, \mathrm{Cl})$ & -0.06 & -0.06 & -0.05 & -0.05 & -0.06 & -0.08 \\
\hline Total & 97.06 & 96.21 & 95.05 & 93.28 & 94.48 & 92.44 \\
\hline \multicolumn{7}{|c|}{ atoms on the basis of $(\mathrm{O})=20,(\mathrm{OH}, \mathrm{F}, \mathrm{Cl})=4$} \\
\hline Si & 5.414 & 5.456 & 5.660 & 5.764 & 5.712 & 5.650 \\
\hline $\mathrm{Al}^{\mathrm{iv}}$ & 2.586 & 2.544 & 2.340 & 2.123 & 2.079 & 2.350 \\
\hline$A d^{v i}$ & 0.186 & 0.205 & 0.037 & - & - & 0.011 \\
\hline $\mathrm{Cr}$ & - & 0.002 & na & na & - & 0.003 \\
\hline V & 0.002 & - & na & na & - & na \\
\hline $\mathrm{Ti}$ & 0.204 & 0.163 & 0.089 & 0.046 & 0.237 & 0.085 \\
\hline $\mathrm{Zr}$ & na & na & 0.001 & 0.006 & na & 0.002 \\
\hline $\mathrm{Fe}$ as $\mathrm{Fe}^{\mathrm{II}}$ & 4.506 & 5.118 & 5.554 & 5.576 & 5.944 & 5.410 \\
\hline $\mathrm{Mn}$ & 0.041 & 0.073 & 0.010 & 0.149 & 0.080 & 0.088 \\
\hline $\mathrm{Zn}$ & 0.014 & 0.017 & na & na & 0.003 & na \\
\hline $\mathrm{Ni}$ & 0.009 & - & na & na & na & - \\
\hline $\mathrm{Mg}$ & 1.001 & 0.470 & 0.281 & 0.482 & 0.042 & 0.537 \\
\hline $\mathrm{Ca}$ & - & 0.004 & 0.008 & 0.026 & 0.013 & 0.015 \\
\hline $\mathrm{Na}$ & 0.222 & 0.198 & 0.247 & 0.183 & 0.081 & 0.146 \\
\hline $\mathrm{K}$ & 1.758 & 1.633 & 1.735 & 1.716 & 1.721 & 1.712 \\
\hline Sr & - & 0.008 & na & na & - & na \\
\hline $\mathrm{Ba}$ & 0.042 & 0.031 & na & na & - & na \\
\hline $\mathrm{F}$ & 0.042 & 0.047 & 0.053 & 0.047 & 0.076 & 0.085 \\
\hline $\mathrm{Cl}$ & 0.035 & 0.032 & 0.011 & 0.013 & 0.004 & 0.022 \\
\hline$(\mathrm{OH})$ & 3.923 & 3.922 & 3.936 & 3.940 & 3.920 & 3.893 \\
\hline cation total & 15.985 & 15.920 & 16.052 & 16.071 & 15.912 & 16.009 \\
\hline $\mathrm{Mg} \#$ & 18.18 & 8.41 & 4.82 & 7.95 & 0.70 & 9.03 \\
\hline
\end{tabular}

na - not analysed; pheno - phenocryst; ind - inclusion; gmass - groundmass; $\mathrm{mt}$ - magnetite; fa - fayalite. 

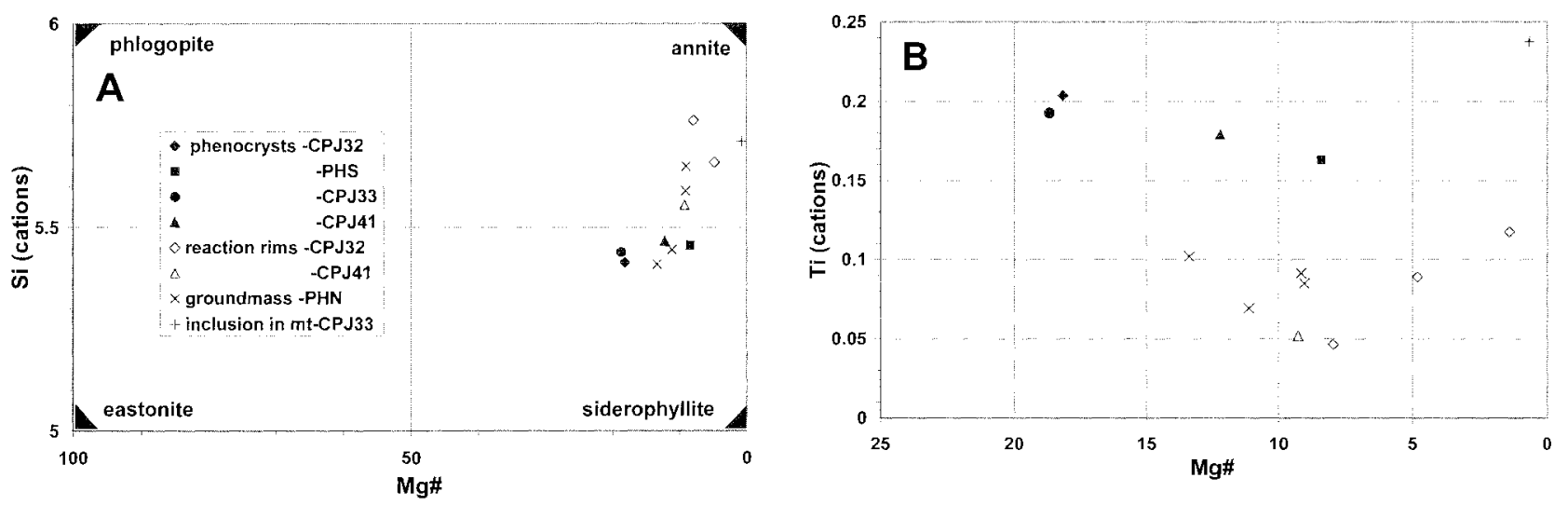

FIG. 5 - Electron microprobe analyses of biotite. (A) Si cations against Mg\#, ideal end-member compositions also shown. (B) Ti cations against $M g \#$.

The biotite microphenocrysts are probably cognate products of fractionating phonolitic magma at depth, and disequilibrium at emplacement may be due to lower pressure. In some samples, biotite re-appeared later as a groundmass phase, probably because crystallisation of sodic amphiboles and pyroxenes had increased $\mathrm{K} / \mathrm{Na}$ in the residual liquid just prior to solidification.

\section{Feldspars}

Both X-ray diffraction and electron microprobe data indicate that two alkali felspars (sanidine and anorthoclase) are present. Simple Carlsbad twinning is ubiquitous and very fine, multiple twinning common, but cross-hatched twinning was not observed. Undulose extinction indicating compositional zoning is common. In some samples, feldspar is turbid and partly altered to fine-grained sericite ( $\mathrm{pl} .3$ ).

Most of the analysed feldspars (table 7, fig. 6) are anorthoclase $\left(\mathrm{Or}_{100-37.6}\right)$ with small amounts of $\mathrm{CaO}$ (up to 8.6 mole \% anorthite in solid solution). Some have significant $\mathrm{BaO}(\leq 2.51 \%)$ and $\mathrm{SrO}(\leq 1.31 \%)$, corresponding to 4.5 mole \% celsian (Ba-feldspar) and 3.4 mole \% Srfeldspar, respectively. The remaining analyses are sanidine $\left(\mathrm{Or}_{62.8-91.1}\right)$ with very low $\mathrm{CaO}, \mathrm{BaO}$ and $\mathrm{SrO}$. All analyses contain minor iron $(0.15-0.31 \%$ as $\mathrm{FeO})$.
The groundmass of CPJ 41 contains small grains of nearstoichiometric celsian, and a grain intermediate between celsian and K-feldspar. Celsian is rare in igneous rocks, and mostly occurs in hydrothermal or contact-metamorphic manganese deposits. Here, it is probably a very late-stage deuteric mineral. Some feldspars plot very close to the albite or K-feldspar end-members, but the analyses are less satisfactory, with high totals and/or significant cation deficiencies. These may have crystallised or been altered during late-stage hydrothermal processes.

\section{Pyroxenes}

Small, well-formed, elongate to acicular prisms (typically 20 -100 to rarely $400 \mu \mathrm{m}$ long $x 5-20 \mu \mathrm{m}$ across) of pale yellow to green clinopyroxene are abundant (pl. 6). Analyses (table 8) are recalculated to four cations and $\mathrm{Fe}$ distributed to $\mathrm{Fe}^{\mathrm{III}}$ and $\mathrm{Fe}^{\text {ll }}$ to ensure charge balance ( 6 oxygens). Although all these pyroxenes are iron-rich with low $\mathrm{TiO}_{2}(\leq 0.43 \%)$ and little or no tetrahedrally coordinated $\mathrm{Al}$, they fall into two groups. The nomenclature follows Morimoto (1988).

Those from CPJ32, and a few from PHN and CPJ33, are relatively calcic (CaO 13.06-19.27\%) and are hedenbergites or relatively calcic aegirine-augites (fig. 7). In CPJ32, their pleochroism is $\alpha$ deep green, $\beta$ pale yellow-green, $\gamma$ pale yellow-brown.

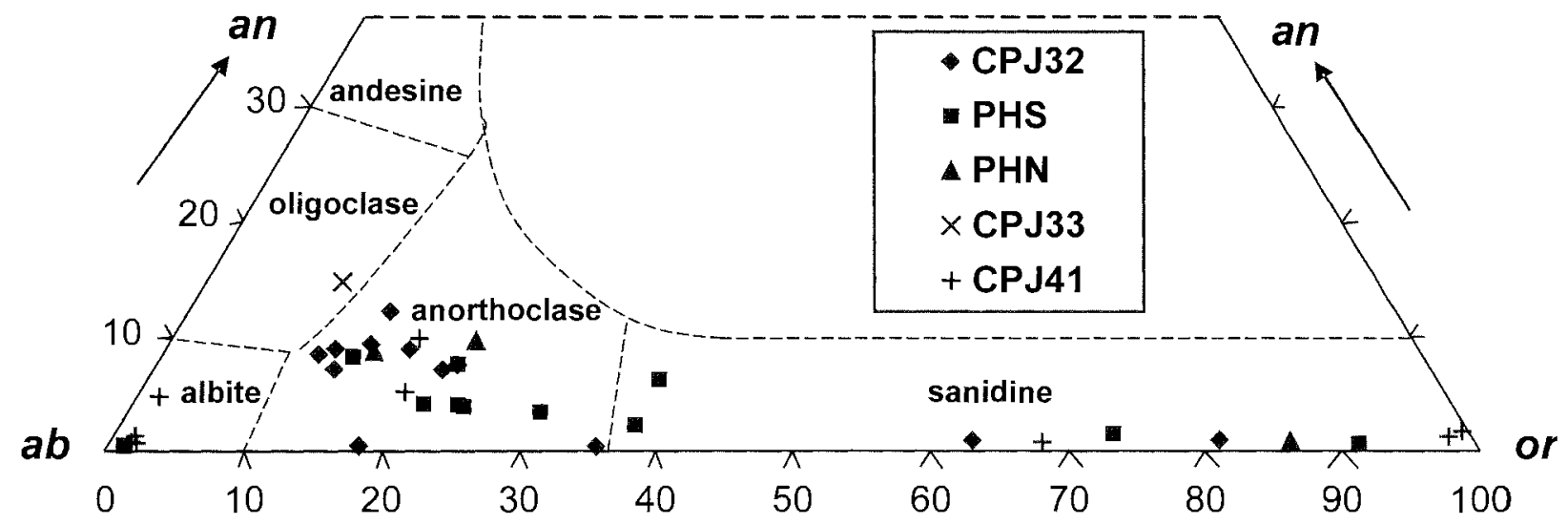

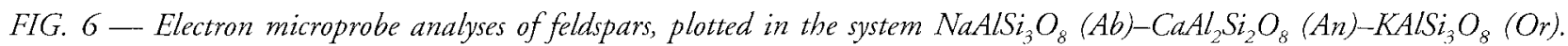
Small amounts of Ba and Sr included with An; celsian (Ba feldspar) analyses not plotted. 
TABLE 7

Representative mineral analyses: feldspars, feldspathoids and analcime

\begin{tabular}{|c|c|c|c|c|c|c|c|c|c|c|}
\hline Sample & CPJ32 & PHS & PHN & CPJ32 & PHS & $\mathrm{PHN}$ & CPJ41 & CPJ32 & PHS & CPJ33 \\
\hline Analysis & $\mathrm{R} 1 / 4$ & $\mathrm{R} 11 / 2$ & $\mathrm{R} 4 / 3$ & $\mathrm{R} 9 / \mathrm{a}$ & $\mathrm{R} 8 / 1$ & $\mathrm{R} 6 / 7$ & el & $\mathrm{R} 11 / 5$ & $\mathrm{R} 12 / 4$ & $\mathrm{R} 1 / 6$ \\
\hline mineral & \multicolumn{3}{|c|}{ anorthoclase } & \multicolumn{3}{|c|}{ sanidine } & celsian & nepheline & sodalite & analcime \\
\hline $\mathrm{SiO}_{2}$ & 66.04 & 64.18 & 63.71 & 65.52 & 63.96 & 63.75 & 31.50 & 43.39 & 37.41 & 56.57 \\
\hline $\mathrm{TiO}_{2}$ & 0.02 & 0.02 & - & 0.02 & - & - & - & - & - & - \\
\hline $\mathrm{Al}_{2} \mathrm{O}_{3}$ & 21.65 & 19.81 & 20.03 & 19.55 & 18.95 & 18.29 & 25.98 & 32.60 & 31.49 & 23.12 \\
\hline $\mathrm{FeO}$ & 0.28 & 0.24 & 0.27 & 0.15 & 0.31 & 0.11 & 0.25 & 0.18 & 0.23 & 0.06 \\
\hline $\mathrm{MgO}$ & - & - & - & - & - & - & 0.01 & - & - & 0.02 \\
\hline $\mathrm{CaO}$ & 1.28 & 0.45 & 0.56 & 0.05 & - & - & 0.19 & - & 0.00 & - \\
\hline $\mathrm{Na}_{2} \mathrm{O}$ & 8.47 & 6.46 & 7.71 & 4.15 & 0.98 & 1.51 & 0.10 & 14.89 & 24.54 & 13.01 \\
\hline $\mathrm{K}_{2} \mathrm{O}$ & 2.03 & 6.46 & 3.81 & 10.79 & 15.69 & 14.60 & 0.05 & 6.48 & 0.10 & 0.08 \\
\hline $\mathrm{P}_{2} \mathrm{O}_{5}$ & - & na & na & 0.02 & - & na & na & na & na & na \\
\hline $\mathrm{SrO}$ & - & 0.40 & 0.76 & 0.01 & - & - & 0.04 & - & - & - \\
\hline $\mathrm{BaO}$ & 1.02 & 1.48 & 2.51 & 0.13 & 0.13 & 0.28 & 40.00 & 0.01 & 0.05 & - \\
\hline $\mathrm{SO}_{3}$ & na & na & na & na & na & na & na & na & 0.40 & na \\
\hline $\mathrm{Cl}$ & - & na & na & - & 0.06 & na & na & na & 6.86 & na \\
\hline $\mathrm{O}=\mathrm{Cl}$ & - & na & na & - & -0.01 & na & na & na & -1.55 & na \\
\hline TOTAL & 100.80 & 99.49 & 99.36 & 100.39 & 100.06 & 98.53 & 98.11 & 97.55 & 99.55 & 92.87 \\
\hline \multicolumn{11}{|c|}{ atoms on the basis of $(\mathrm{O})=$} \\
\hline & 8 & 8 & 8 & 8 & 8 & 8 & 8 & 4 & 12.5 & 6 \\
\hline Si & 2.911 & 2.929 & 2.913 & 2.964 & 2.963 & 2.986 & 2.013 & 1.064 & 3.026 & 2.035 \\
\hline $\mathrm{Ti}$ & 0.001 & 0.001 & - & 0.001 & - & - & - & - & - & - \\
\hline $\mathrm{Al}$ & 1.125 & 1.066 & 1.079 & 1.043 & 1.035 & 1.001 & 1.957 & 0.942 & 3.001 & 0.980 \\
\hline $\mathrm{Fe}$ & 0.010 & 0.009 & 0.010 & 0.006 & 0.012 & 0.004 & 0.013 & 0.004 & 0.015 & 0.002 \\
\hline $\mathrm{Mg}$ & - & - & - & - & - & - & 0.001 & - & - & 0.001 \\
\hline $\mathrm{Ca}$ & 0.061 & 0.022 & 0.028 & 0.002 & - & - & 0.013 & - & 0.001 & - \\
\hline $\mathrm{Na}$ & 0.724 & 0.571 & 0.683 & 0.364 & 0.088 & 0.137 & 0.012 & 0.708 & 3.848 & 0.907 \\
\hline $\mathrm{K}$ & 0.114 & 0.376 & 0.222 & 0.623 & 0.927 & 0.872 & 0.004 & 0.203 & 0.011 & 0.004 \\
\hline $\mathrm{Sr}$ & - & 0.011 & 0.020 & - & - & - & 0.001 & - & - & - \\
\hline $\mathrm{Ba}$ & 0.018 & 0.026 & 0.045 & 0.002 & 0.002 & 0.005 & 1.002 & - & 0.002 & - \\
\hline P & - & na & na & 0.001 & - & na & na & na & na & na \\
\hline$S$ & na & na & na & na & na & na & na & na & 0.024 & na \\
\hline $\mathrm{Cl}$ & - & na & na & - & 0.004 & na & na & na & 0.940 & na \\
\hline cation total & 4.963 & 5.011 & 5.000 & 5.006 & 5.027 & 5.014 & 5.017 & 2.920 & 9.903 & 3.930 \\
\hline \multicolumn{11}{|c|}{ end-members (mole \%) } \\
\hline an & 6.6 & 2.2 & 2.8 & 0.2 & - & - & 1.2 & ne 71.7 & & \\
\hline $\mathrm{ab}$ & 79.0 & 56.8 & 68.4 & 36.7 & 8.6 & 13.5 & 1.2 & ks 20.5 & & \\
\hline or & 12.4 & 37.4 & 22.3 & 62.8 & 91.1 & 86.0 & 0.4 & Q 7.8 & & \\
\hline Sr feldspar & - & 1.1 & 2.0 & - & - & - & 0.1 & & & \\
\hline celsian & 1.9 & 2.6 & 4.5 & 0.2 & 0.2 & 0.5 & 97.0 & & & \\
\hline
\end{tabular}


TABLE 8

Representative mineral analyses: pyroxenes

\begin{tabular}{|c|c|c|c|c|c|c|}
\hline Sample & CPJ32 & $\mathrm{PHN}$ & PHN & CPJ33 & PHS & CPJ33 \\
\hline Analysis & $\mathrm{R} 6 / 1$ & $\mathrm{R} 7 / 2$ & $\mathrm{R} 4 / 5$ & $\mathrm{R} 7 / 3$ & $\mathrm{R} 2 / 3$ & $\mathrm{R} 2 / 4$ \\
\hline Mineral & \multicolumn{2}{|c|}{ Hedenbergite } & \multicolumn{2}{|c|}{ Aegirine-augite } & \multicolumn{2}{|c|}{ Aegirine } \\
\hline $\mathrm{SiO}_{2}$ & 46.90 & 48.05 & 49.35 & 50.64 & 52.05 & 51.49 \\
\hline $\mathrm{TiO}_{2}$ & 0.32 & 0.12 & 0.16 & 0.41 & 0.20 & 0.34 \\
\hline $\mathrm{ZrO}_{2}$ & 0.35 & 0.28 & 0.51 & 1.19 & 0.46 & 2.11 \\
\hline $\mathrm{Al}_{2} \mathrm{O}_{3}$ & 1.99 & 1.42 & 2.23 & 1.20 & 3.88 & 1.03 \\
\hline $\mathrm{Cr}_{2} \mathrm{O}_{3}$ & 0.03 & - & 0.02 & - & 0.01 & - \\
\hline $\mathrm{eO}_{\mathrm{t}}$ & 24.72 & 24.90 & 26.58 & 27.70 & 25.38 & 27.57 \\
\hline $\operatorname{lnO}$ & 1.02 & 1.03 & 0.90 & 0.63 & 0.57 & 0.32 \\
\hline $\mathrm{iO}$ & - & 0.03 & - & - & & 0.04 \\
\hline $\mathrm{MgO}$ & .75 & 2.14 & 0.50 & 0.01 & 0.17 & 0.29 \\
\hline $\mathrm{aO}$ & 19.01 & 19.15 & 13.16 & 6.56 & 2.19 & 2.92 \\
\hline $\mathrm{a}_{2} \mathrm{O}$ & .21 & 2.06 & .59 & 9.37 & 12.01 & 11.40 \\
\hline $\mathrm{K}_{2} \mathrm{O}$ & & 0.02 & 0.02 & - & 0.05 & 0.03 \\
\hline Total & 98.54 & 99.19 & 99.00 & 97.79 & 96.98 & 97.55 \\
\hline \multicolumn{7}{|c|}{ Cations calculated on the basis of $Z=4,(\mathrm{O})=6$} \\
\hline $\mathrm{Si}$ & 1.899 & 1.934 & 1.953 & 1.986 & 1.989 & 1.998 \\
\hline $\mathrm{Al}^{\mathrm{iv}}$ & 0.095 & 0.066 & 0.047 & 0.014 & 0.011 & 0.002 \\
\hline $\mathrm{Al}^{\mathrm{vi}}$ & - & 0.001 & 0.057 & 0.042 & 0.164 & 0.046 \\
\hline & 0.001 & 0.004 & 0.005 & 0.012 & & 0.001 \\
\hline $\mathrm{Zr}$ & 0.007 & 0.006 & 0.001 & 0.023 & 0.009 & 0.040 \\
\hline $\mathrm{Cr}$ & 0.001 & - & 0.001 & - & 0.000 & - \\
\hline $\mathrm{Fe}^{\mathrm{III}}$ & 0.253 & 0.209 & 0.389 & 0.615 & 0.711 & 0.716 \\
\hline $\mathrm{Fe}^{\mathrm{II}}$ & 0.584 & 0.629 & 0.491 & 0.293 & 0.100 & 0.179 \\
\hline $\mathrm{Mn}$ & 0.035 & 0.035 & 0.030 & 0.021 & 0.018 & 0.011 \\
\hline 101 & - & 0.001 & - & - & - & 0.001 \\
\hline $\mathrm{Mg}$ & 0.105 & 0.128 & 0.029 & 0.006 & 0.001 & 0.017 \\
\hline $\mathrm{Ca}$ & 0.829 & 0.826 & 0.558 & 0.276 & 0.090 & 0.122 \\
\hline $\mathrm{Na}$ & 0.174 & 0.161 & 0.429 & 0.713 & 0.890 & 0.858 \\
\hline & 0.008 & 0.001 & 0.000 & - & 0.002 & 0.001 \\
\hline $\mathrm{Mg \#}$ & 15.30 & 16.92 & 5.64 & 1.90 & 9.01 & 8.66 \\
\hline
\end{tabular}

The remaining analyses, including all from $\mathrm{PHN}$ and CPJ41, are sodic pyroxenes with low $\mathrm{CaO}(\leq 7.12 \%)$. Most are aegirines, but a few are sodic aegirine-augites. Crystallographic orientation is difficult due to the small grain size, but in PHS pleochroism is approximately $\alpha$ pale green, $\beta$ and $\gamma$ pale greenish yellow.

$\mathrm{MnO}(\leq 1.19 \%)$ ranges to higher values in hedenbergite and calcic aegirine-augite, whereas $\mathrm{Al}_{2} \mathrm{O}_{3}$ (1.03-3.88\%) ranges higher in aegirine, implying some solid solution towards jadeite. $\mathrm{ZrO}_{2}$ is variable but sometimes relatively high (0.28-0.54\% in hedenbergite, $0.10-2.11 \%$ in aegirine) and does not correlate with other elements, grain size or morphology.

An apparent slight excess of $\mathrm{Si}(\leq 2.027$ cations per formula unit) in some aegirine analyses may result from small amounts of unanalysed elements. In particular, Nb is relatively high in the whole rock (169-174 ppm, see

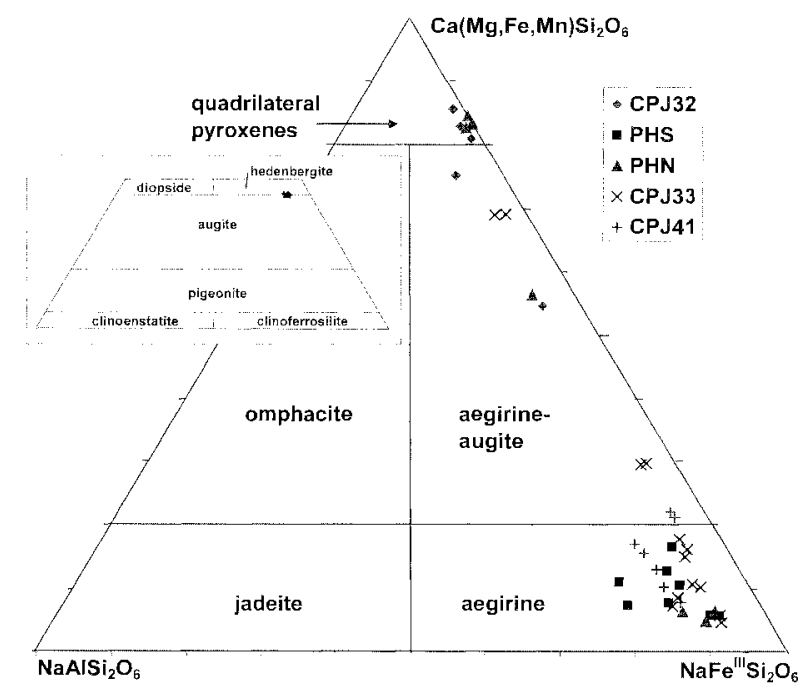

FIG. 7 - Electron microprobe analyses of pyroxenes projected on to the plane $\mathrm{NaAlSi}_{2} \mathrm{O}_{6}$ (jadeite)-NaFe $\mathrm{Ni}_{2} \mathrm{O}_{6}$ (acmite) $\mathrm{Ca}(\mathrm{Mg}, \mathrm{Fe}, \mathrm{Mn}) \mathrm{Si}_{2} \mathrm{O}_{6}$ (quadrilateral clinopyroxene). Inset shows hedenbergites plotted on to the pyroxene quadrilateral (diopsidehedenbergite-clinoenstatite-clinoferrosilite). Nomenclature after Morimoto (1988).

below) and can enter sodic pyroxenes, as Nickel \& Mark (1965) report $1.04 \% \mathrm{Nb}_{2} \mathrm{O}_{5}$ in aegirine-augite (and $0.14 \%$ in arfvedsonite) in a paragneiss at Seal Lake, Labrador.

\section{Amphiboles}

Sodic amphibole is common in the groundmass as ragged prisms (mostly $\leq 100 \mu \mathrm{m}$ long) (pl. 5) and more rarely as subhedral to euhedral pseudohexagonal sections $(\leq 200 \mu \mathrm{m}$ across) (pl. 6) or glomerocrystic clots. In PHS, an amphibole rhomb $(200 \times 100 \mu \mathrm{m})$ encloses a fayalite grain $(70 \mu \mathrm{m})$.

Analyses (table 9) were converted to the amphibole formula $\mathrm{AB}_{2} \mathrm{C}_{5} \mathrm{~T}_{8} \mathrm{O}_{22}(\mathrm{OH}, \mathrm{F}, \mathrm{Cl})_{2}$ by the method of $\mathrm{J}$. C. Schumacher (in Leake 1997, appendix 2). For all of these amphiboles, estimates of minimum $\mathrm{Fe}^{\mathrm{III}}$ are given by the "16CAT" formulae, in which the cations are recalculated to sum to 16 , implying full occupancy of the A sites, and Fe distributed between $\mathrm{Fe}^{\mathrm{III}}$ and $\mathrm{Fe}^{\mathrm{II}}$ to maintain charge balance. They fall into two distinct populations.

Those from CPJ32 and a few from CPJ33 are iron-rich calcic amphiboles with substantial substitution of $\mathrm{Al}$ for $\mathrm{Si}$ in the tetrahedral (T) sites. They also have low $\mathrm{Mg} /\left(\mathrm{Mg}+\mathrm{Fe}^{\mathrm{II}}\right)$, and since $\mathrm{Fe}{ }^{\mathrm{III}}>\mathrm{Al}$ in the $\mathrm{C}$ sites most fall into the hastingsite field (fig. 8). Analysis R7/5 falls into the ferroedenite field because of its slightly higher Si. Optically, the hastingsite in CPJ32 is length slow with distinctive pleochroism ( $\alpha$ pale straw yellow-brown, $\beta$ deep khaki green to olive-green, $\gamma$ blue-green).

The remaining analyses, including all those from PHS, PHN and CPJ41, have much higher $\mathrm{SiO}_{2}$ and $\mathrm{Na}_{2} \mathrm{O}$ and very low $\mathrm{Al}_{2} \mathrm{O}_{3}$ and $\mathrm{CaO}$. They are sodic amphiboles, because the $\mathrm{B}$ sites are largely occupied by $\mathrm{Na}$. Because of their low tetrahedral $\mathrm{Al}$ and high $\mathrm{Mg} / \mathrm{Mg}+\mathrm{Fe}^{\mathrm{II}}$ and $\mathrm{Fe}^{\mathrm{III}}$ relative to octahedral Al, they are classified as arfvedsonite (fig. 8), and several lie close to the end-member $(\mathrm{Na}, \mathrm{K}) \cdot \mathrm{Na}_{2} \cdot \mathrm{Fe}_{4}{ }^{\mathrm{II}} \mathrm{Fe}{ }^{\mathrm{III}}$. $\mathrm{Si}_{8} \mathrm{O}_{22}(\mathrm{OH}, \mathrm{F})_{2}$. Optically, the atfvedsonite in PHS is length fast and strongly coloured ( $\alpha$ very dark blue-green, $\beta$ pale greenish- to olive-brown, $\gamma$ dark grey-green). 
TABLE 9

Representative mineral analyses: amphiboles

\begin{tabular}{|c|c|c|c|c|c|c|c|}
\hline \multirow{3}{*}{\multicolumn{2}{|c|}{$\begin{array}{l}\text { Sample } \\
\text { Analysis } \\
\text { Mineral }\end{array}$}} & CPJ32 & CPJ33 & \multirow{2}{*}{$\begin{array}{c}\text { CPJ32 } \\
\text { R7/5 }\end{array}$} & PHS & \multirow{2}{*}{$\begin{array}{l}\mathrm{CPJ} 33 \\
\mathrm{R} 2 / 2\end{array}$} & \multirow{2}{*}{$\begin{array}{l}\text { CPJ } 41 \\
\mathrm{R} 1 / 7\end{array}$} \\
\hline & & $\mathrm{R} 2 / 3$ & $\mathrm{R} 4 / 1$ & & $\mathrm{R} 7 / 2$ & & \\
\hline & & \multicolumn{2}{|c|}{ Hastingsite } & Edenite & \multicolumn{3}{|c|}{ Arfvedsonite } \\
\hline \multicolumn{2}{|c|}{$\mathrm{SiO}_{2}$} & 38.66 & 39.05 & 40.58 & 49.03 & 48.19 & 47.94 \\
\hline \multicolumn{2}{|c|}{$\mathrm{TiO}_{2}$} & 0.67 & 0.69 & 0.52 & 0.04 & 0.51 & 0.14 \\
\hline \multicolumn{2}{|c|}{$\mathrm{ZrO}_{2}$} & 0.28 & 0.52 & 0.33 & 0.04 & 0.13 & 2.08 \\
\hline \multicolumn{2}{|c|}{$\mathrm{Al}_{2} \mathrm{O}_{3}$} & 9.11 & 8.43 & 8.09 & 1.04 & 1.45 & 1.77 \\
\hline \multicolumn{2}{|c|}{$\mathrm{Cr}_{2} \mathrm{O}_{3}$} & 0.04 & 0.01 & - & 0.04 & - & 0.04 \\
\hline \multicolumn{2}{|c|}{$\mathrm{FeO}$} & 30.49 & 32.18 & 31.05 & 31.87 & 32.17 & 32.69 \\
\hline \multicolumn{2}{|c|}{$\mathrm{MnO}$} & 0.77 & 0.74 & 0.78 & 1.83 & 1.68 & 1.64 \\
\hline \multicolumn{2}{|l|}{$\mathrm{NiO}$} & - & - & - & - & - & 0.03 \\
\hline \multicolumn{2}{|c|}{$\mathrm{MgO}$} & 2.47 & 1.61 & 1.91 & 2.21 & 0.87 & 0.73 \\
\hline \multicolumn{2}{|c|}{$\mathrm{CaO}$} & 9.96 & 9.97 & 8.84 & 1.29 & 0.76 & 0.83 \\
\hline \multicolumn{2}{|l|}{$\mathrm{SrO}$} & - & - & - & 0.21 & - & 0.06 \\
\hline \multicolumn{2}{|c|}{$\mathrm{Na}_{2} \mathrm{O}$} & 3.18 & 3.21 & 3.51 & 6.57 & 7.70 & 7.56 \\
\hline \multicolumn{2}{|l|}{$\mathrm{K}_{2} \mathrm{O}$} & 1.24 & 1.42 & 1.44 & 2.92 & 2.07 & 2.15 \\
\hline \multicolumn{2}{|l|}{$\mathrm{BaO}$} & - & - & 0.09 & - & - & 0.04 \\
\hline \multicolumn{2}{|l|}{$\mathrm{F}$} & 0.07 & 0.09 & 0.07 & 0.23 & 0.22 & 0.25 \\
\hline \multicolumn{2}{|l|}{$\mathrm{Cl}$} & 0.06 & 0.05 & 0.06 & - & 0.02 & 0.01 \\
\hline \multicolumn{2}{|c|}{$\mathrm{O}=(\mathrm{F}, \mathrm{Cl})$} & -0.04 & -0.05 & -0.04 & -0.01 & -0.01 & -0.11 \\
\hline Total & & 96.96 & 97.93 & 97.24 & 97.23 & 95.67 & 97.86 \\
\hline atom & son $t$ & the basis & of $\mathrm{AB}_{2} \mathrm{C}_{5}$ & $\mathrm{~T}_{8} \mathrm{O}_{22}(\mathrm{Ol}$ & $\mathrm{I}, \mathrm{F})_{2}(16$ & CAT form & hulae) \\
\hline Si & $\mathrm{T}$ & 6.291 & 6.348 & 6.605 & 7.859 & 7.841 & 7.720 \\
\hline $\mathrm{Al}^{\mathrm{iv}}$ & $\mathrm{T}$ & 1.709 & 1.615 & 1.395 & 0.141 & 0.159 & 0.280 \\
\hline $\mathrm{Al}^{\mathrm{vi}}$ & $\mathrm{C}$ & 0.038 & - & 0.156 & 0.056 & 0.119 & 0.056 \\
\hline $\mathrm{Cr}$ & $\mathrm{C}$ & 0.005 & 0.002 & - & 0.005 & - & 0.005 \\
\hline $\mathrm{Ti}$ & $\mathrm{C}$ & 0.082 & 0.084 & 0.064 & 0.005 & 0.063 & 0.017 \\
\hline $\mathrm{Zr}$ & $\mathrm{C}$ & 0.022 & 0.041 & 0.026 & 0.003 & 0.010 & 0.164 \\
\hline $\mathrm{Fe}^{1.1 \mathrm{I}}$ & $\mathrm{C}$ & 0.720 & 0.744 & 0.466 & 0.704 & 0.752 & 0.659 \\
\hline $\mathrm{Fe}^{\mathrm{II}}$ & $\mathrm{C}$ & 3.429 & 3.630 & 3.761 & 3.568 & 3.624 & 3.744 \\
\hline $\mathrm{Ni}$ & C & - & - & - & - & - & 0.004 \\
\hline $\mathrm{Mg}$ & $\mathrm{C}$ & 0.600 & 0.390 & 0.464 & 0.529 & 0.212 & 0.176 \\
\hline $\mathrm{Mn}$ & C & 0.104 & 0.102 & 0.063 & 0.130 & 0.221 & 0.176 \\
\hline Mn & B & 0.002 & - & 0.045 & 0.119 & 0.011 & 0.048 \\
\hline $\mathrm{Ca}$ & B & 1.736 & 1.737 & 1.542 & 0.222 & 0.132 & 0.143 \\
\hline Sr & B & - & - & - & 0.020 & - & 0.005 \\
\hline $\mathrm{Na}$ & B & 0.262 & 0.307 & 0.413 & 1.640 & 1.857 & 1.804 \\
\hline $\mathrm{Na}$ & A & 0.742 & 0.705 & 0.695 & 0.402 & 0.571 & 0.555 \\
\hline K & A & 0.258 & 0.295 & 0.299 & 0.598 & 0.429 & 0.442 \\
\hline $\mathrm{Ba}$ & A & - & - & 0.006 & - & - & 0.002 \\
\hline $\mathrm{F}$ & & 0.035 & 0.045 & 0.037 & 0.116 & 0.113 & 0.129 \\
\hline $\mathrm{Cl}$ & & 0.016 & 0.015 & 0.015 & - & 0.005 & 0.003 \\
\hline $\mathrm{OH}$ & & 1.949 & 1.941 & 1.957 & 1.884 & 1.883 & 1.868 \\
\hline $\mathrm{Mg} \#$ & & 14.89 & 9.70 & 10.98 & 12.91 & 5.52 & 4.49 \\
\hline
\end{tabular}

Arfvedsonite is lower in $\mathrm{TiO}_{2}$ and higher in $\mathrm{MnO}$ $(\leq 2.95 \%)$ and $\mathrm{K}_{2} \mathrm{O}(\leq 3.10 \%)$ than hastingsite. All are low in $\mathrm{Ba}, \mathrm{Sr}, \mathrm{F}(\leq 0.25 \%)$ and $\mathrm{Cl}(\leq 0.06 \%)$. As in pyroxenes, $\mathrm{ZrO}_{2}$ is very variable but sometimes considerable (up to $2.08 \%$ in arfvedsonite).

For some analyses, the total occupancy of the $\mathrm{T}$ and $\mathrm{C}$ sites is slightly less than 13 , due to an excess of cations that must be allocated to the $\mathrm{A}$ and $\mathrm{B}$ sites (i.e., $\mathrm{Ca}, \mathrm{Na}$, $\mathrm{Ba}$ and $\mathrm{Sr}$ ) relative to all other cations. This cannot be corrected by any assumption regarding $\mathrm{Fe}^{\mathrm{III}} / \mathrm{Fe}^{\mathrm{II}}$, and suggests an analytical problem. It is possible that there are small amounts of additional, unanalysed elements in the $\mathrm{C}$ sites, particularly REE or $\mathrm{Nb}$.

The formulae can be projected on to a ternary diagram (fig. 8) depicting the three mutually coupled substitutions $S$ i $\leftrightarrow \mathrm{Al}$ in the $\mathrm{T}$ sites, $\left(\mathrm{Mg}, \mathrm{Fe}^{\mathrm{II}}, \mathrm{Mn}\right) \leftrightarrow\left(\mathrm{Al}, \mathrm{Fe}^{\mathrm{III}}\right)$ in the $\mathrm{C}$ sites, and $\mathrm{Na} \leftrightarrow \mathrm{Ca}$ in the $\mathrm{B}$ sites. The amphiboles lie fairly close to the end members of the join between hastingsitel pargasite and arfvedsonite/eckermannite, in which the first and third of the above substitutions are coupled.

\section{Apatite}

Accessory apatite is present as small laths $(\leq 250 \times 20 \mu \mathrm{m})$, acicular prisms (pl. 6) and hexagonal cross-sections (rarely $\leq 100 \mu \mathrm{m}$ but mostly $\leq 15 \mu \mathrm{m}$ ) in the groundmass and as inclusions in fayalite and magnetite microphenocrysts.

Typical analyses (table 10) are near-stoichiometric, with mostly small amounts of iron $(\leq 1.29 \%$ as $\mathrm{FeO}), \mathrm{SrO}$ $(\leq 0.94 \%), \mathrm{BaO}(\leq 0.96 \%), \mathrm{Na}_{2} \mathrm{O}(\leq 0.53 \%)$ and light rare earth elements (LREE) $\left(\leq 1.65 \% \mathrm{La}_{2} \mathrm{O}_{3}+\mathrm{Ce}_{2} \mathrm{O}_{3}\right)$. They are relatively low in $\mathrm{F}$ (one analysis $1.88 \%$, rest $\leq 1.18 \%$ ) and $\mathrm{Cl}(\leq 0.37 \%)$ and are mostly hydroxyapatites.

Small apatite grains from CPJ 41 and PHS, however, have large amounts of $\mathrm{SrO}(\leq 25.74 \%)$ and LREE $(\leq 12.22 \%)$. In CPJ4 1 they accompany celsian and may be a late stage hydrothermal mineral.

\section{Nepheline, Sodalite and Analcime}

The feldspathoidal material consists of an irregularly polygonal aggregate of colourless anhedra, each typically $25-150 \mu \mathrm{m}$ across. Some is weakly birefringent and sometimes slightly turbid, and has optical properties consistent with nepheline. The remainder, with slightly higher relief, is clear and isotropic (pl. 3), and probably consists of sodalite and analcime, as indicated by X-ray diffraction. The more altered samples (CPJ33, PHN) lack nepheline and sodalite, and analcime is more abundant.

Because of their fine grain size, "clean" near-stoichiometric microprobe analyses of these minerals (table 7) were difficult to obtain, and some are probably mixtures of two or more $\mathrm{Na}-\mathrm{Al}$ silicates and other minerals. In addition, the analyses are usually deficient in $\mathrm{Na}$ relative to the ideal formulae, probably due to volatilisation in the electron beam. Small amounts of iron (usually $<0.5 \%$ as $\mathrm{FeO}$ ) are invariably present.

Reasonably stoichiometric analyses of nepheline were obtained from CPJ32, PHS and CPJ41. They contain appreciable $\mathrm{K}_{2} \mathrm{O}(4.67-6.69 \%)$ suggesting approximately $15-20$ mole $\% \mathrm{KAlSiO}_{4}$ in solid solution, and "excess silica" corresponding to $10-15 \%$ solid solution towards alkali feldspar. Near-stoichiometric sodalite $\left(\mathrm{Na}_{4} \mathrm{Al}_{3} \mathrm{Si}_{3} \mathrm{O}_{8} \mathrm{Cl}\right)$ was 


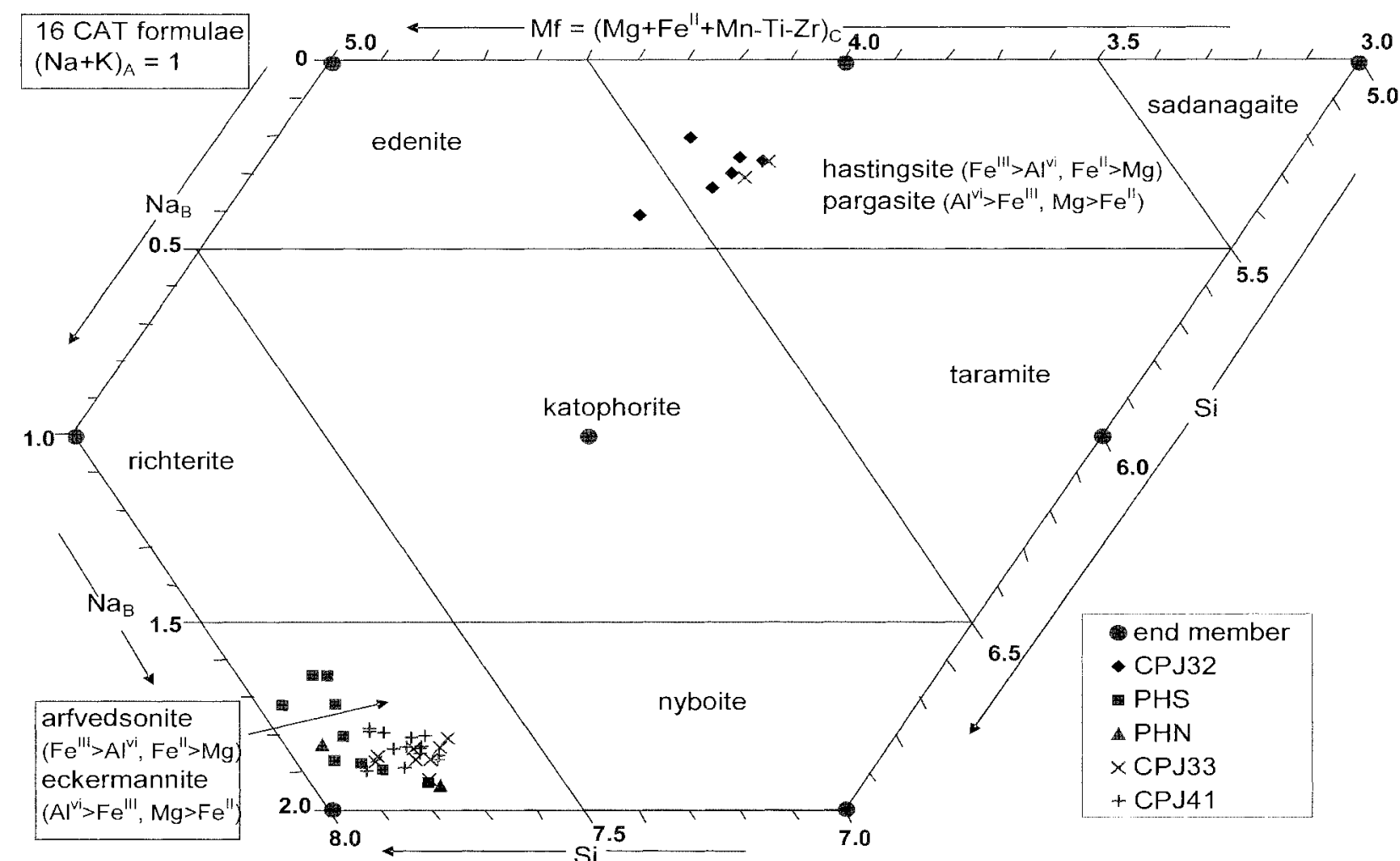

FIG. 8 - Electron microprobe analyses of amphiboles (16 CAT, minimum Fe formulae) projected on to the system edeniterichterite-arfvedsoniteleckermannite-nyboite-taramite-hastingsitelpargasite, showing coupled substitutions and ideal end-member compositions. Nomenclature consistent with Leake et al. (1997). See text for further discussion.

analysed from CPJ32 and PHS. In contrast to nepheline, $\mathrm{K}_{2} \mathrm{O}$ is negligible.

Possible analcime analyses, from four samples, are mostly less stoichiometric. Those from CPJ32 and PHS contain significant iron and/or $\mathrm{K}_{2} \mathrm{O}$, and some have excess alkalis suggesting contamination by sodalite.

\section{DISCUSSION}

\section{Phenocrysts}

The rare fayalite, biotite and magnetite phenocrysts probably represent near-liquidus phases that crystallised from the phonolitic magma at depth. They may have buffered $\mathrm{fO}_{2}$ by reactions of the form

$$
\text { [1a] } 3 \mathrm{Fe}_{2} \mathrm{SiO}_{4}+\mathrm{O}_{2}=2 \mathrm{Fe}_{3} \mathrm{O}_{4}+3 \mathrm{SiO}_{2}
$$

This is the well-known quartz-fayalite-magnetite buffer, but its $\mathrm{T}_{-} \mathrm{fO}_{2}$ calibration is not directly applicable, as $\mathrm{SiO}_{2}$ is a low-activity component in the melt, rather than a mineral phase. Alternatively, it could be rewritten as

$$
\begin{aligned}
{[\mathrm{lb}] 6 \mathrm{Fe}_{2} \mathrm{SiO}_{4}+3(\mathrm{Na}, \mathrm{K}) \mathrm{AlSiO}_{4}+2 \mathrm{O}_{2}=} \\
4 \mathrm{Fe}_{3} \mathrm{O}_{4}+3(\mathrm{Na}, \mathrm{K}) \mathrm{AlSi}_{3} \mathrm{O}_{8}
\end{aligned}
$$

where nepheline, kalsilite (K-nepheline) and probably alkali feldspar are components in the melt, rather than mineral phases. Although alkali feldspar occurs as phenocrysts in the phonolite, they grade down into the groundmass and probably crystallised later than the other phenocrysts.
The strong resorption of biotite and fayalite phenocrysts may in part be due to adiabatic decompression, but the general absence of these minerals in the groundmass suggests that they were not stable at the lower pressures and temperatures during final emplacement and cooling of the dyke. Fayalite and magnetite are frequently surrounded by a reaction rim of biotite, which may be represented by reactions of the form:

$$
\begin{aligned}
& {[2 \mathrm{a}] 6 \mathrm{Fe}_{2} \mathrm{SiO}_{4}+4 \mathrm{KAlSiO}_{4}+} 2 \mathrm{SiO}_{2}+4 \mathrm{H}_{2} \mathrm{O}= \\
& 4 \mathrm{KFe}_{3} \mathrm{AlSi}_{3} \mathrm{O}_{10}(\mathrm{OH})_{2} \\
& {[3 \mathrm{a}] 2 \mathrm{Fe}_{3} \mathrm{O}_{4}+2 \mathrm{KAlSiO}_{4}+} 4 \mathrm{SiO}_{2}+2 \mathrm{H}_{2} \mathrm{O}= \\
& 2 \mathrm{KFe}_{3} \mathrm{AlSi}_{3} \mathrm{O}_{10}(\mathrm{OH})_{2}+\mathrm{O}_{2}
\end{aligned}
$$

or

$$
\begin{array}{r}
{[2 \mathrm{~b}] 6 \mathrm{Fe}_{2} \mathrm{SiO}_{4}+3 \mathrm{KAlSiO}_{4}+\mathrm{KAISi}_{3} \mathrm{O}_{8}+4 \mathrm{H}_{2} \mathrm{O}=} \\
4 \mathrm{KFe}_{3} \mathrm{AlSi}_{3} \mathrm{O}_{10}(\mathrm{OH})_{2}
\end{array}
$$

[3b] $2 \mathrm{Fe}_{3} \mathrm{O}_{4}+2 \mathrm{KAlSi}_{3} \mathrm{O}_{8}+2 \mathrm{H}_{2} \mathrm{O}=$

$$
2 \mathrm{KFe}_{3} \mathrm{AlSi}_{3} \mathrm{O}_{10}(\mathrm{OH})_{2}+\mathrm{O}_{2}
$$

It is clear from reactions [2] and [3], which intersect with [1] (fig. 9), that increasing $\mathrm{fH}_{2} \mathrm{O}$ (or decreasing temperature) will favour biotite relative to fayalite and magnetite respectively. From [3], lower $\mathrm{fO}_{2}$ will also favour biotite relative to magnetite. The experiments of Wones $\&$ Eugster (1965) place [3b] for pure annite (Fe-biotite) at about $680^{\circ} \mathrm{C}$ at $103.5 \mathrm{MPa}$, or about $710^{\circ} \mathrm{C}$ at $207 \mathrm{MPa}$, for oxygen fugacities controlled by the quartz-fayalitemagnetite buffer [1a]. 
TABLE 10

Representative mineral analyses: apatite

\begin{tabular}{|c|c|c|}
\hline Sample & CPJ 41 & CPJ41 \\
\hline Analysis & a5 & a7 \\
\hline $\mathrm{SiO}_{2}$ & 0.33 & 2.79 \\
\hline $\mathrm{ThO}_{2}$ & 0.01 & 0.68 \\
\hline $\mathrm{La}_{2} \mathrm{O}_{3}$ & 0.40 & 5.02 \\
\hline $\mathrm{Ce}_{2} \mathrm{O}_{3}$ & 0.64 & 5.92 \\
\hline $\mathrm{Pr}_{2} \mathrm{O}_{3}$ & 0.13 & 0.37 \\
\hline $\mathrm{Nd}_{2} \mathrm{O}_{3}$ & 0.24 & 0.91 \\
\hline $\mathrm{Sm}_{2} \mathrm{O}_{3}$ & - & 0.02 \\
\hline $\mathrm{Y}_{2} \mathrm{O}_{3}$ & 0.08 & 0.04 \\
\hline $\mathrm{FeO}$ & 0.46 & 0.65 \\
\hline $\mathrm{MnO}$ & 0.15 & 0.19 \\
\hline $\mathrm{MgO}$ & 0.02 & 0.01 \\
\hline $\mathrm{CaO}$ & 52.72 & 20.52 \\
\hline $\mathrm{SrO}$ & 1.16 & 25.74 \\
\hline $\mathrm{BaO}$ & - & 0.81 \\
\hline $\mathrm{Na}_{2} \mathrm{O}$ & 0.27 & 0.64 \\
\hline $\mathrm{P}_{2} \mathrm{O}_{5}$ & 40.62 & 31.70 \\
\hline $\mathrm{As}_{2} \mathrm{O}_{5}$ & 0.04 & 0.01 \\
\hline $\mathrm{SO}_{3}$ & 0.01 & 0.02 \\
\hline $\mathrm{F}$ & 1.03 & 2.03 \\
\hline $\mathrm{Cl}$ & 0.32 & 0.01 \\
\hline $\mathrm{O}=(\mathrm{F}, \mathrm{Cl})$ & -0.51 & -0.86 \\
\hline TOTAL & 98.13 & 97.23 \\
\hline \multicolumn{3}{|c|}{ Atoms on the basis of $(\mathrm{O})=12,(\mathrm{OH}, \mathrm{F}, \mathrm{Cl})=1$} \\
\hline $\mathrm{P}$ & 2.954 & 2.838 \\
\hline As & 0.002 & 0.001 \\
\hline S & 0.001 & 0.001 \\
\hline Si & 0.028 & 0.296 \\
\hline Th & - & 0.016 \\
\hline $\mathrm{La}$ & 0.013 & 0.196 \\
\hline $\mathrm{Ce}$ & 0.020 & 0.229 \\
\hline $\operatorname{Pr}$ & 0.004 & 0.014 \\
\hline Nd & 0.008 & 0.035 \\
\hline $\operatorname{Sm}$ & - & 0.001 \\
\hline Y & 0.003 & 0.002 \\
\hline $\mathrm{Fe}^{\mathrm{II}}$ & 0.033 & 0.057 \\
\hline $\mathrm{Mn}$ & 0.011 & 0.017 \\
\hline $\mathrm{Mg}$ & 0.002 & 0.001 \\
\hline $\mathrm{Ca}$ & 4.852 & 2.324 \\
\hline $\mathrm{Sr}_{\mathrm{r}}$ & 0.058 & 1.578 \\
\hline $\mathrm{Ba}$ & - & 0.034 \\
\hline $\mathrm{Na}$ & 0.045 & 0.131 \\
\hline $\mathrm{F}$ & 0.279 & 0.678 \\
\hline $\mathrm{Cl}$ & 0.047 & 0.003 \\
\hline$(\mathrm{OH})$ & 0.674 & 0.319 \\
\hline cation total & 8.034 & 7.771 \\
\hline
\end{tabular}

\section{Groundmass}

Fergusson (1978) noted that a compositional gap occurs between $\mathrm{Ca}$ - and $\mathrm{Na}$ - rich pyroxenes within single samples of many felsic alkaline rocks, and attributed this to crystallisation of amphibole, particularly in slowly cooled intrusive rocks. In the Tomahawk phonolite, most pyroxenes are either hedenbergite or aegirine, but the analyses (fig. 7) do not show a clear compositional gap. Those in PHN and CPJ33 have a wide range with some intermediate compositions (aegirineaugite). As both pyroxene and amphibole are groundmass phases, they probably crystallised together rapidly.

However, a large and obvious compositional gap lies between $\mathrm{Ca}$-rich and $\mathrm{Na}$-rich amphiboles (hastingsite and arfvedsonite), even within a thin section (CPJ33; fig. 8). Intermediate sodic-calcic amphiboles near katophorite $\mathrm{Na}_{\mathrm{A}} \cdot \mathrm{Na}_{\mathrm{B}} \mathrm{Ca}\left(\mathrm{Mg}, \mathrm{Fe}^{\mathrm{II}}\right)_{4}\left(\mathrm{Al}, \mathrm{Fe}^{\mathrm{III}}\right) \cdot \mathrm{AlSi}_{7} \mathrm{O}_{22}(\mathrm{OH}, \mathrm{F}, \mathrm{Cl})_{2}$, on the hastingsite-arfvedsonite compositional join, are absent. Katophorite, which contains both $\mathrm{Na}$ and $\mathrm{Ca}$ in the $\mathrm{B}$ sites, is a comparatively rare high temperature amphibole mainly occurring in ultramafic alkaline rocks (Deer et al. 1997). There may be a miscibility gap between hastingsite and arfvedsonite at the lower temperatures at which the phonolite groundmass crystallised. As the remaining melt cooled and became further depleted in $\mathrm{CaO}$, this solvus may have been intersected, causing the precipitating amphibole to change suddenly from hastingsite to arfvedsonite.

Arfvedsonite and aegirine may be related by reactions of the form

$$
\begin{aligned}
& \text { [4] } 5 \mathrm{NaFe}^{\mathrm{III}} \mathrm{Si}_{2} \mathrm{O}_{6}+\mathrm{H}_{2} \mathrm{O}= \\
& \mathrm{Na}_{3} \mathrm{Fe}_{4}^{\mathrm{II}} \mathrm{Fe}^{\mathrm{III}} \mathrm{Si}_{8} \mathrm{O}_{22}(\mathrm{OH})_{2}+\mathrm{Na}_{2} \mathrm{Si}_{2} \mathrm{O}_{5}+\mathrm{O}_{2} \\
& \text { [5] } 18 \mathrm{NaFe}^{\mathrm{III}} \mathrm{Si}_{2} \mathrm{O}_{6}+4 \mathrm{Fe}_{3} \mathrm{O}_{4}+12 \mathrm{SiO}_{2}+6 \mathrm{H}_{2} \mathrm{O}= \\
& 6 \mathrm{Na}_{3} \mathrm{Fe}_{4}{ }_{4} \mathrm{Fe}^{1 \mathrm{III}} \mathrm{Si}_{8} \mathrm{O}_{22}(\mathrm{OH})_{2}+5 \mathrm{O}_{2}
\end{aligned}
$$

where $\mathrm{SiO}_{2}$ and $\mathrm{Na}_{2} \mathrm{Si}_{2} \mathrm{O}_{5}$ are components in the melt, the latter expressing peralkalinity.

These reactions probably intersect (fig. 9) and define a restricted sector in $\mathrm{fO}_{2}-\mathrm{fH}_{2} \mathrm{O}$ space in which both arfvedsonite and aegirine are stable, and magnetite is absent. Clearly, the reduced hydrous phase arfvedsonite is favoured by low $\mathrm{fO}_{2}$ and high $\mathrm{fH}_{2} \mathrm{O}$ (or lower temperature), whereas aegirine is favoured by high $\mathrm{fO}_{2}$ and low $\mathrm{fH}_{2} \mathrm{O}$.

Marsh (1975) suggested that the stability of acmite (end-member aegirine) in strongly undersaturated liquids is limited by the reaction

$$
\text { [6] } 12 \mathrm{NaFe}^{\mathrm{III}} \mathrm{Si}_{2} \mathrm{O}_{6}=4 \mathrm{Fe}_{3} \mathrm{O}_{4}+6 \mathrm{Na}_{2} \mathrm{Si}_{2} \mathrm{O}_{5}+12 \mathrm{SiO}_{2}+\mathrm{O}_{2}
$$

for which thermodynamic calculations suggested unrealistically high oxygen fugacities (above the hematite-magnetite buffer). This is contrary to petrographic evidence in some nepheline syenites (and the Tomahawk phonolite) and was attributed to inaccurate thermodynamic data (specifically, the free energy of acmite).

Aenigmatite $\left(\mathrm{Na}_{2} \mathrm{Fe}_{5}{ }_{5}^{\mathrm{II}} \mathrm{TiSi}_{6} \mathrm{O}_{20}\right)$ is common in many peralkaline phonolites and its absence in the Tomahawk River dyke requires comment. Marsh (1975) noted that it typically forms by the reaction of Ti-magnetite with a peralkaline undersaturated liquid. Because of inadequate thermodynamic data he could not define its stability field, but suggested that it lay at low $\mathrm{fO}_{2}$ and was enlarged by high activity of $\mathrm{Na}_{2} \mathrm{Si}_{2} \mathrm{O}_{5}$ (in melt) and low activity of $\mathrm{NaFeSi}_{2} \mathrm{O}_{6}$ (in pyroxene). High $\mathrm{fO}_{2}$ and/or aegirine-rich 


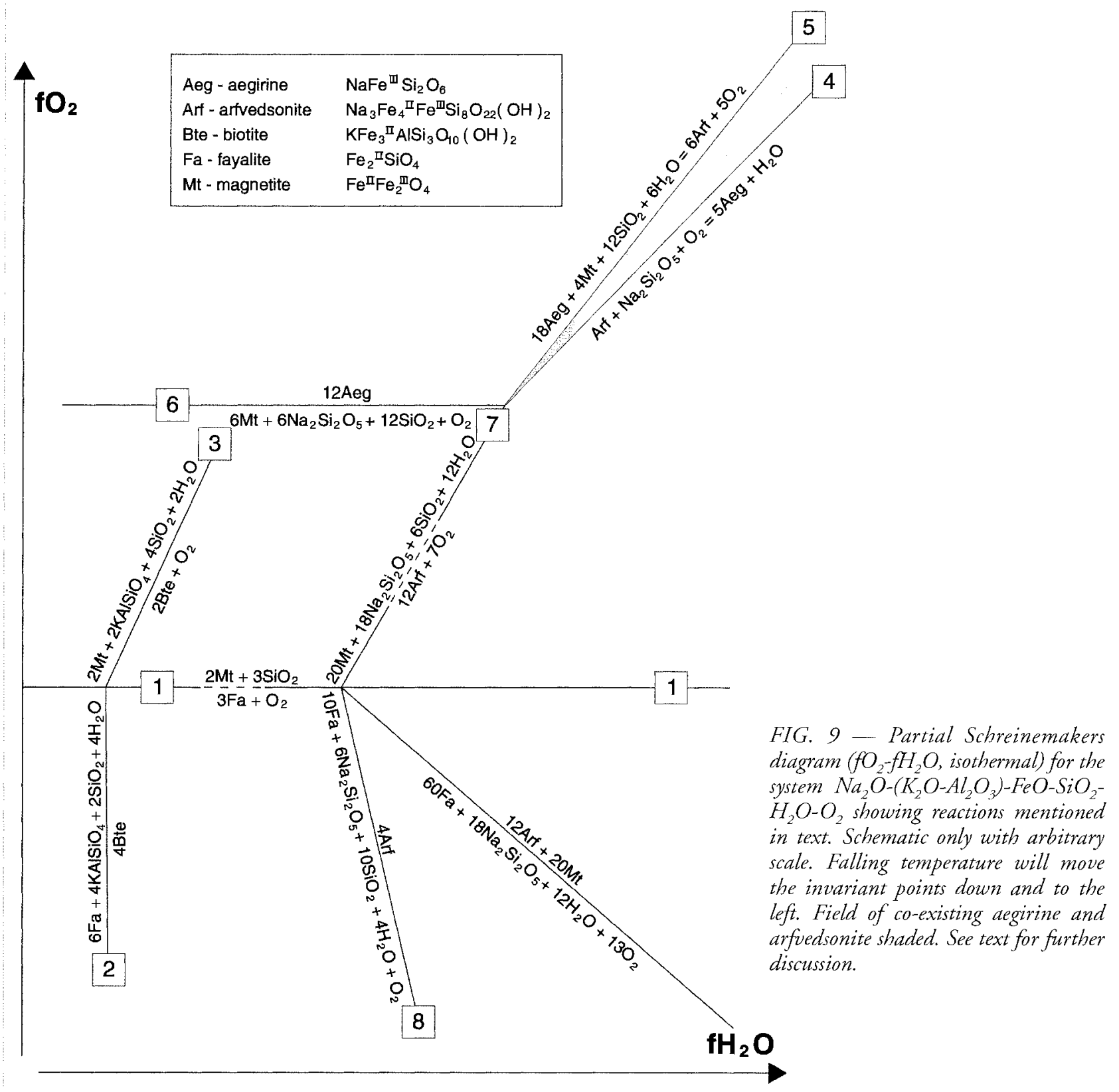

pyroxene may explain the absence of aenigmatite in the Tomahawk River dyke. Alternatively, it may result from the very low $\mathrm{TiO}_{2}$ content $(0.05-0.06 \%$, see below) of the dyke. Although low-Ti aenigmatite has been both synthesised and reported in natural rocks, its stability field is restricted to lower $\mathrm{fO}_{2}$ and pressures than normal aenigmatite (Deer et al. 1997 and references therein). In this rock, reaction of magnetite with peralkaline melt instead produced rims of biotite or haloes of aegirine and arfvedsonite, by reactions [3], [6] and/or

[7] $20 \mathrm{Fe}_{3} \mathrm{O}_{4}+18 \mathrm{Na}_{2} \mathrm{Si}_{2} \mathrm{O}_{5}+6 \mathrm{SiO}_{2}+12 \mathrm{H}_{2} \mathrm{O}=$

$$
12 \mathrm{Na}_{3} \mathrm{Fe}_{4}{ }_{4}^{\mathrm{Il}} \mathrm{Fe}^{\mathrm{III}} \mathrm{Si}_{8} \mathrm{O}_{22}(\mathrm{OH})_{2}+7 \mathrm{O}_{2}
$$

There is also petrographic evidence (pl. 6) for direct reaction of fayalite with peralkaline melt to produce arfvedsonite:

[8] $10 \mathrm{Fe}_{2} \mathrm{SiO}_{4}+6 \mathrm{Na}_{2} \mathrm{Si}_{2} \mathrm{O}_{5}+10 \mathrm{SiO}_{2}+4 \mathrm{H}_{2} \mathrm{O}+\mathrm{O}_{2}=$ $4 \mathrm{Na}_{3} \mathrm{Fe}_{4}^{\mathrm{II}} \mathrm{Fe}^{\mathrm{III}} \mathrm{Si}_{8} \mathrm{O}_{22}(\mathrm{OH})_{2}$

\section{WHOLE ROCK CHEMISTRY}

\section{Analytical Techniques}

Two fresh samples, CPJ32 and PHS, were crushed in a steel jaw crusher. Major elements were determined by X-ray fluorescence (XRF) on glass discs, prepared by standard techniques at Mineral Resources Tasmania (MR'T) laboratories, Hobart. $\mathrm{FeO} / \mathrm{Fe}_{2} \mathrm{O}_{3}$ was determined by titration and $\mathrm{CO}_{2}$ by gravimetric methods. $\mathrm{H}_{2} \mathrm{O}+$ was calculated from loss-onignition after adjusting for $\mathrm{FeO}, \mathrm{CO}_{2}$ and $\mathrm{SO}_{3}$.

Four separate determinations were made of many trace elements. XRF analyses, using standard techniques on pressed pellets, were done at both MRT and the University of Tasmania. Each sample was also analysed by inductively coupled plasma mass spectroscopy (ICP-MS) at the University. Small $(5-10 \mathrm{~mm})$ chips remaining from the initial jaw crushing were ground in agate and digested by two techniques: $\mathrm{HF}-\mathrm{HNO}_{3}$ digestion in Savillex $\mathrm{B}$ teflon vessels on a hotplate at $130^{\circ}-150^{\circ} \mathrm{C}$ and 1 atmosphere, and 
the Pico'Trace@ $\mathrm{HF} / \mathrm{H}_{2} \mathrm{SO}_{4}-\mathrm{HClO}_{4}$ digestion system at $\leq 2000 \mathrm{kPa}$ and $\leq 180^{\circ} \mathrm{C}$. Full details of procedures are given by $\mathrm{Yu}$ et al. (2001) and data by Everard (2004).

Although the ICP-MS data mostly have better precision and lower detection limits than XRF, errors may arise from incomplete digestion of resistant minerals. Data obtained using the more intensive Pico Trace $($ digestion system are generally preferred, as values for several elements (REE, Y, Zr, $\mathrm{Hf}$ and $\mathrm{Sn}$ ) exceed those obtained by Savillex $(\mathrm{R}$ digestion, but are similar to XRF data where available.

For $\mathrm{Cr}, \mathrm{V}$ and $\mathrm{Ba}$, the university XRF data are preferred because of contamination or digestion problems with ICPMS. W and As were below XRF detection limits (10 ppm) at MRT. For $\mathrm{Li}$ and $\mathrm{Be}$, which are difficult to determine, the average of two ICP-MS determinations, rounded to the nearest ppm, are quoted. The remaining trace element data in table 11 were determined by PicoTrace $\mathbb{R}$ digestion and ICP-MS.

\section{Major Elements}

Both analyses are phonolites according to the total alkali-silica diagram of Le Bas et al. (1986) (fig. 10) and the CIPW-normative classification of Johnson \& Duggan (1989) (DI > 75; $A n<50 \mathrm{~mol} \%$, and $n e>10 \%$ ).

The high (>1) Agpaitic Index (table 11) indicates a peralkaline composition (molar $\left(\mathrm{Na}_{2} \mathrm{O}+\mathrm{K}_{2} \mathrm{O}\right)>$ molar $\mathrm{Al}_{2} \mathrm{O}_{3}$ ). Mineralogically, this is expressed by the presence of sodic amphiboles, sodic pyroxenes and sodalite and the absence of calcic plagioclase.

Compared with the average phonolite of Le Maitre (1976), and those in eastern Australia (table 11) and elsewhere, the Tomahawk River phonolite is exceptionally depleted in $\mathrm{TiO}_{2}$, $\mathrm{MgO}$ and $\mathrm{CaO}$ and has unusually high $\mathrm{Na}_{2} \mathrm{O} / \mathrm{K}_{2} \mathrm{O}$. Of the eastern Australian examples quoted, only the Cretaceous (72 Ma) Baralaba plug (Sutherland et al. 1996) is also peralkaline, although less strongly so, and more depleted in $\mathrm{MgO}, \mathrm{CaO}$ and also $\mathrm{P}_{2} \mathrm{O}_{5}$.

When CIPW-normative components $o r, a b$ and ne are calculated and plotted as weight \% on to the $\mathrm{NaAlSiO}_{4-}$ $\mathrm{KAlSiO}_{4}-\mathrm{SiO}_{2}$ system (fig. 11), the analyses plot very near the feldspar-nepheline temperature minima as determined at atmospheric pressure (Schairer 1950) and $98 \mathrm{MPa}(0.98 \mathrm{~kb}$ ) water pressure (Hamilton \& McKenzie 1965). This suggests that, like many other phonolites, compositions were largely controlled by crystal-liquid equilibrium processes at relatively low pressures. However, the minima in this system are relatively insensitive to pressure, and the Tomahawk River phonolite has other significant components, particularly the $\mathrm{Na}$-bearing component $a c$ (acmite) which accounts for its peralkalinity and high $\mathrm{Na}_{2} \mathrm{O} / \mathrm{K}_{2} \mathrm{O}$.

Bailey (1976) reviewed experimental work relevant to peralkaline feldspathoidal rocks. Although the effect of acmite (end-member aegirine) on the K-bearing system is not well determined, Schairer \& Bowen (1956) found that excess $\mathrm{Na}_{2} \mathrm{O}$ (i.e., peralkalinity) at 1 atmosphere extends the albite-nepheline cotectic from $1063^{\circ} \mathrm{C}$ to a eutectic with $\mathrm{Na}_{2} \mathrm{Si}_{2} \mathrm{O}_{5}$ at $732^{\circ} \mathrm{C}$. Bailey \& Schairer (1966) found that the phase relations in system $\mathrm{Na}_{2} \mathrm{O}-\mathrm{Al}_{2} \mathrm{O}_{3}-\mathrm{Fe}_{2} \mathrm{O}_{3}-\mathrm{SiO}_{2}$ at 1 atmosphere largely reflect the incongruent melting of acmite (to hematite and liquid). Crystallisation of albite, nepheline and acmite from a sodium silicate (ns)-normative liquid leads to a eutectic with $\mathrm{Na}_{2} \mathrm{Si}_{2} \mathrm{O}_{5}$ at $715^{\circ} \pm 5^{\circ} \mathrm{C}$. This matches the late groundmass crystallisation of aegirine in the Tomahawk River phonolite. Nolan (1966) found that the minimum $\left(715^{\circ} \mathrm{C}\right.$ at $\left.98 \mathrm{MPa}\right)$ in the join albite-nephelineacmite coexists with magnetite and a very iron-rich liquid, but that the addition of minor diopside greatly enlarges the stability field of pyroxene.

\section{Trace Elements}

In common with most phonolites, the Tomahawk River dyke is strongly depleted in trace elements $(\mathrm{Ni}, \mathrm{Cr}, \mathrm{Sc}, \mathrm{V}$, $\mathrm{Co}$ ) that are compatible in early crystallising ferromagnesian minerals. Both high field strength elements (HFSE) ( Nb, Zr, $\mathrm{La}, \mathrm{Ce})$ and $\mathrm{Rb}$ are high, as found in many phonolites and intermediate-silicic Cainozoic and Mesozoic volcanic rocks from eastern Australia (e.g., Ewart et al. 1985, Sutherland et al. 1996).

Published analyses of phonolites (table 11 and reference list) show extreme variability in Sr (e.g., 5-3000 ppm) and $\mathrm{Ba}$ (e.g., 2-1645 ppm). The Tomahawk River dyke is relatively high in $\mathrm{Sr}(430,570 \mathrm{ppm})$ and especially Ba (1040, $1300 \mathrm{ppm}$ ).

The chondrite-normalised REE plots (fig. 12) have distinctive concave patterns, with strong light REE (LREE) enrichment, relative middle REE (MREE) depletion, and a flat to slightly positive slope in heavy REE (HREE). Both analyses have very slight positive Eu anomalies, in contrast to the negative Eu anomaly of the $\mathrm{Mt}$ Wilson phonolite in Victoria (Ewart et al. 1985). Only a few published phonolite REE data display negative Eu anomalies, and those rocks, like the $\mathrm{Mt}$ Wilson phonolite, are commonly also low in $\mathrm{Sr}$ and Ba (e.g., Weaver 1990, Ablay et al. 1998). At Cantal, Massif Central (France), Wilson et al. (1995) distinguished aphyric "Type A" phonolites with low $\mathrm{Sr}, \mathrm{Ba}$ and negative Eu anomalies from porphyritic "Type B" phonolites lacking these features.

Mantle-normalised spidergrams (fig. 13) illustrate the very strong depletions in $\mathrm{TiO}_{2}$ and $\mathrm{P}_{2} \mathrm{O}_{5}$, whereas tetravalent trace elements ( $\mathrm{Zr}, \mathrm{Hf}, \mathrm{Sn}$ ) together with $\mathrm{Pb}, \mathrm{Mo}, \mathrm{Sb}$ and $\mathrm{Li}$ are enriched relative to comparable REE. These features presumably reflect fractionation processes rather than characteristics of the mantle source: $\mathrm{Ti}$ and $\mathrm{P}$ have clearly strongly partitioned into a fractionating phase,

\section{Key to Table 11 (opposite)}

atomic $100 \mathrm{Mg} /\left(\mathrm{Mg}+\mathrm{Fe}^{\mathrm{II}}\right)$

atomic $100 \mathrm{Mg} /\left(\mathrm{Mg}+\mathrm{Fe}^{\text {rocal }}\right)$

agpaitic (peralkaline) index: molar $\left(\mathrm{Na}_{2} \mathrm{O}+\mathrm{K}_{2} \mathrm{O}\right) / \mathrm{Al}_{2} \mathrm{O}_{3}$

mean phonolite, $\mathrm{n}=340$ (Le Maitre, 1976)

2 "phonolite" [trachyte], 5.92 Ma, sample 7809/3, Mt Wilson, Trentham, Vic (Ewart et al., 1985)

3 "phonolite" [trachyte], Jurassic, sample 7809/10, Coleraine, W Vic (Ewart et al., 1985)

4 phonolite, $169 \mathrm{Ma}$, sample 7809/25, Pinnacle Mt, via Mullaley, NSW (Ewart et al., 1985)

5 nepheline syenite plug, $72 \mathrm{Ma}$, Mt Ramsay, via Baralaba, Qld (Sutherland et al., 1996)

'phonolite, <2.8 Ma, Phonolite Hill, McBride province, N Qld (Stephenson et al., 1980) 
TABLE 11

Whole-rock analyses

Tomahawk River World Av $\quad$ East Australian phonolites and similar rocks

\begin{tabular}{|c|c|c|c|c|c|c|c|c|}
\hline Field No. & CPJ32 & PHS & 1 & 2 & 3 & 4 & 5 & 6 \\
\hline $\mathrm{SiO}_{2}(\%)$ & 56.23 & 56.75 & 56.19 & 59.39 & 59.04 & 56.57 & 61.80 & 54.34 \\
\hline $\mathrm{TiO}_{2}$ & 0.06 & 0.05 & 0.62 & 0.13 & 0.10 & 0.14 & 0.12 & 0.14 \\
\hline $\mathrm{Al}_{2} \mathrm{O}_{3}$ & 18.99 & 19.01 & 19.04 & 18.36 & 18.61 & 19.51 & 18.20 & 19.46 \\
\hline $\mathrm{Fe}_{2} \mathrm{O}_{3}$ & 3.27 & 3.86 & 2.79 & 3.78 & 2.67 & 3.54 & 3.10 & 4.15 \\
\hline $\mathrm{FeO}$ & 3.01 & 2.24 & 2.03 & 1.93 & 2.57 & 2.34 & 1.10 & 1.55 \\
\hline $\mathrm{MnO}$ & 0.19 & 0.20 & 0.17 & 0.13 & 0.19 & 0.17 & 0.14 & 0.17 \\
\hline $\mathrm{MgO}$ & 0.26 & 0.17 & 1.07 & 0.35 & 0.31 & 0.37 & 0.10 & 0.98 \\
\hline $\mathrm{CaO}$ & 1.01 & 0.83 & 2.72 & 1.25 & 1.36 & 1.72 & 0.50 & 1.89 \\
\hline $\mathrm{Na}_{2} \mathrm{O}$ & 10.46 & 10.32 & 7.79 & 7.51 & 6.80 & 8.08 & 8.30 & 8.18 \\
\hline $\mathrm{K}_{2} \mathrm{O}$ & 4.03 & 3.95 & 5.24 & 5.46 & 5.68 & 5.40 & 4.90 & 4.46 \\
\hline $\mathrm{P}_{2} \mathrm{O}_{5}$ & 0.25 & 0.19 & 0.18 & 0.08 & 0.04 & 0.16 & 0.01 & 0.29 \\
\hline $\mathrm{SO}_{3}$ & 0.09 & 0.02 & nd & nd & nd & nd & nd & nd \\
\hline $\mathrm{CO}_{2}$ & 0.02 & 0.11 & 0.08 & - & 0.19 & 0.27 & $<0.10$ & 0.14 \\
\hline $\mathrm{H}_{2} \mathrm{O}+$ & 1.56 & 1.32 & 1.57 & 0.70 & 1.75 & 0.97 & 1.21 & 1.34 \\
\hline $\mathrm{H}_{2} \mathrm{O}-$ & nd & nd & 0.37 & 0.24 & 0.70 & 0.68 & 0.38 & 1.60 \\
\hline Total & 99.42 & 99.01 & 99.41 & 99.31 & 100.01 & 99.92 & 99.96 & 98.71 \\
\hline $\mathrm{FeO}^{\text {rocal }}$ & 5.95 & 5.71 & 4.54 & 5.33 & 4.97 & 5.53 & 3.89 & 5.28 \\
\hline $\mathrm{Mg \#}^{a}$ & 13.20 & 11.92 & 48.44 & 24.43 & 17.79 & 21.99 & 13.95 & 52.99 \\
\hline $\mathrm{mg \#}{ }^{b}$ & 7.14 & 5.04 & 29.58 & 10.48 & 10.01 & 10.66 & 4.38 & 24.85 \\
\hline $\mathrm{AI}^{\mathrm{c}}$ & 1.136 & 1.118 & 0.971 & 0.995 & 0.931 & 0.981 & 1.042 & 0.940 \\
\hline Li (ppm) & 32 & 50 & & & & & & \\
\hline $\mathrm{Be}$ & 7 & 9 & & & & & & \\
\hline$S_{C}$ & 3.99 & 1.26 & & 0.97 & & & & \\
\hline $\mathrm{V}$ & 15.8 & $<1.5$ & & 10 & 15 & 15 & 2 & \\
\hline $\mathrm{Cr}$ & 14.1 & 3.3 & & 3 & 2 & & $<10$ & 34 \\
\hline Co & 4.36 & 1.04 & & & & & & \\
\hline $\mathrm{Ni}$ & 7.27 & 0.64 & & 4 & 5 & 3 & $<10$ & 8 \\
\hline $\mathrm{Cu}$ & 12.3 & 6.0 & & 10 & 19 & 12 & $<5$ & 13 \\
\hline $\mathrm{Zn}_{\mathrm{n}}$ & 193 & 219 & & 255 & 148 & 171 & & 125 \\
\hline Ga & 30.2 & 32.4 & & & & & 41 & 27 \\
\hline $\mathrm{Rb}$ & 127 & 131 & & 261 & 241 & 126 & 195 & 36 \\
\hline $\mathrm{Sr}$ & 572 & 434 & & 41 & 20 & 129 & 12 & 848 \\
\hline$Y$ & 22.4 & 24.7 & & 43 & 45 & 25 & 125 & 20 \\
\hline $\mathrm{Zr}_{\mathrm{r}}$ & 758 & 917 & & 1710 & 842 & 932 & 1485 & 965 \\
\hline $\mathrm{Nb}$ & 169 & 194 & & 283 & 228 & 145 & 210 & 236 \\
\hline Mo & 10.4 & 6.0 & & & & & & \\
\hline $\mathrm{Sn}$ & 6.13 & 7.20 & & & & & & \\
\hline $\mathrm{Sb}$ & 0.24 & 0.27 & & 0.64 & & & & \\
\hline $\mathrm{Cs}$ & 3.17 & 3.20 & & 8.4 & & & & \\
\hline $\mathrm{Ba}$ & 1304 & 1041 & & 40 & 19 & 174 & 120 & 128 \\
\hline $\mathrm{La}$ & 86.8 & 99.0 & & 137 & & & 141 & 66 \\
\hline $\mathrm{Ce}$ & 136.9 & 149.2 & & 224 & 251 & 138 & 270 & 116 \\
\hline $\operatorname{Pr}$ & 13.2 & 13.8 & & & & & 32 & \\
\hline $\mathrm{Nd}$ & 38.9 & 40.2 & & 58 & & & 94 & \\
\hline $\mathrm{Sm}$ & 5.69 & 5.84 & & 8.3 & & & 17 & \\
\hline $\mathrm{Eu}$ & 1.82 & 1.78 & & 0.85 & & & & \\
\hline $\mathrm{Gd}$ & 4.28 & 4.35 & & & & & 16 & \\
\hline $\mathrm{Tb}$ & 0.70 & 0.72 & & 1.12 & & & & \\
\hline Dy & 4.07 & 4.38 & & & & & 17 & \\
\hline Ho & 0.81 & 0.87 & & 1.44 & & & & \\
\hline $\mathrm{Er}$ & 2.39 & 2.65 & & & & & & \\
\hline $\mathrm{Tm}$ & 0.39 & 0.42 & & 0.63 & & & & \\
\hline $\mathrm{Yb}$ & 2.49 & 2.72 & & 4.63 & & & 5 & \\
\hline I_u & 0.40 & 0.43 & & 0.68 & & & & \\
\hline $\mathrm{Hf}$ & 14.9 & 17.7 & & 36.6 & & & 18 & \\
\hline $\mathrm{Ta}$ & 11.5 & 13.0 & & 20.5 & & & & \\
\hline $\mathrm{Tl}$ & 0.60 & 0.68 & & & & & & \\
\hline $\mathrm{Pb}$ & 20.2 & 17.6 & & 25 & 23 & 15 & 27 & 12 \\
\hline $\mathrm{Bi}$ & 0.09 & 0.10 & & & & & & \\
\hline Th & 20.4 & 27.7 & & 58 & 46 & 19 & 30 & 21 \\
\hline $\mathrm{U}$ & 5.74 & 7.53 & & 8.9 & & & 11 & 2 \\
\hline
\end{tabular}




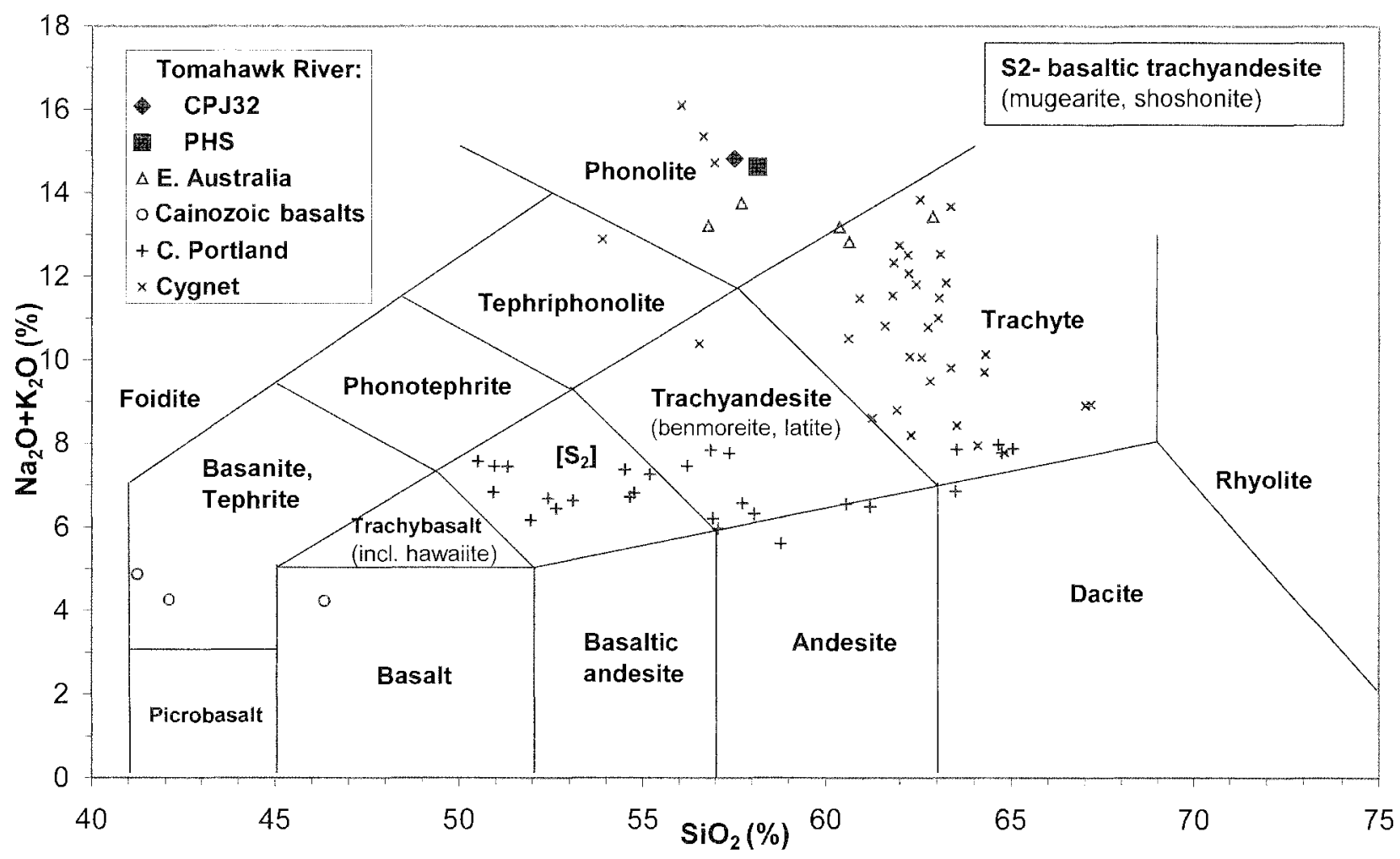

FIG. 10 - Whole-rock analyses of the Tomahawk phonolite plotted on the total alkali-silica diagram, showing volcanic rock nomenclature of Le Bas et al. (1986). Data from other eastern Australian phonolites and trachytes (table 9), local Cainozoic basalts and Cape Portland appinites (J. L. Everard unpublished data) and Cygnet syenites (Taheri \& Bottrill 1999) shown for comparison. All data recalculated to $100 \%$ anhydrous.

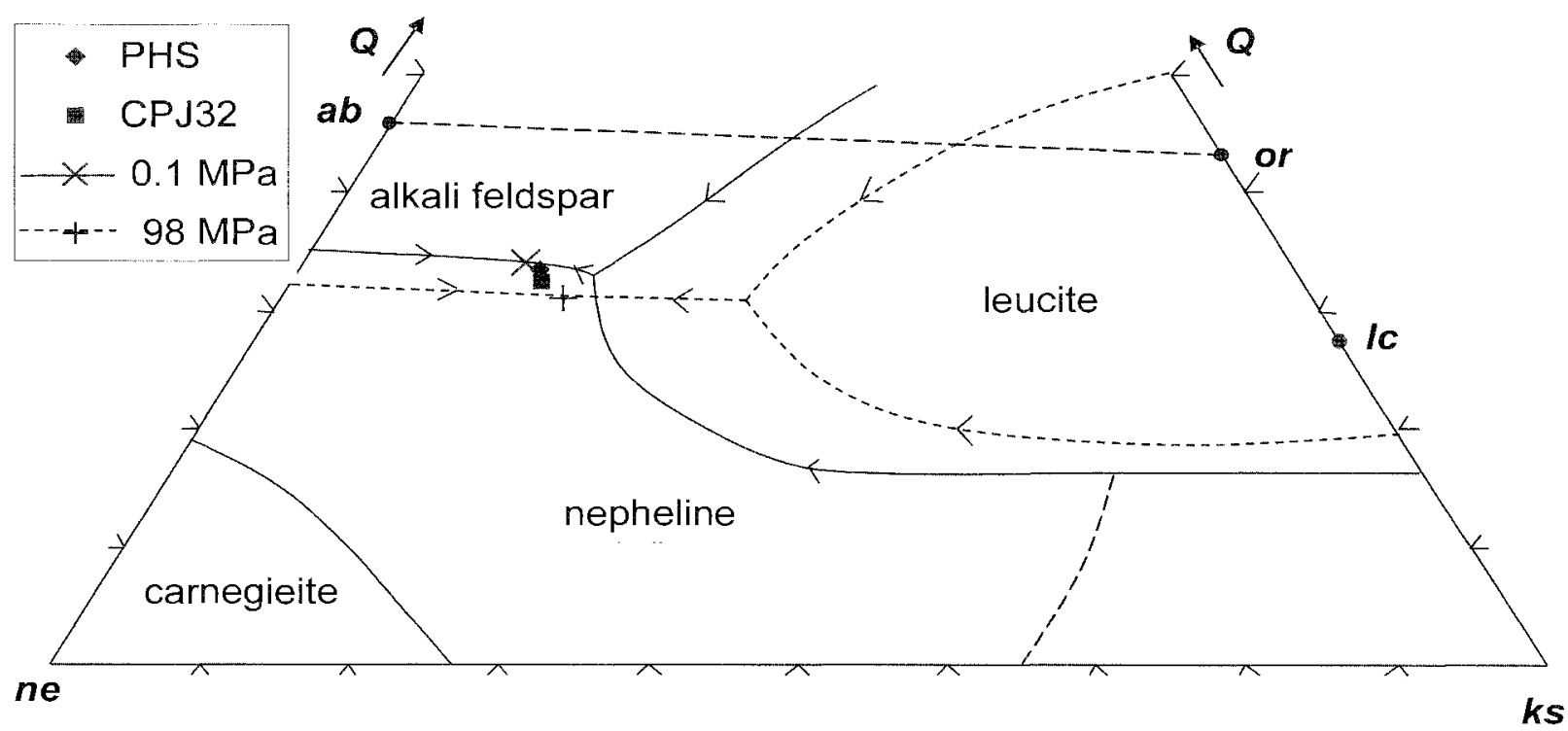

FIG. 11 - Whole-rock analyses of the Tomahawk phonolite projected on to part of the system $\mathrm{NaAlSiO}_{4}-\mathrm{KALISO}_{4}-\mathrm{SiO}_{2}$, showing experimentally determined phase relations at $0.1 \mathrm{MPa}$ (Schairer 1950) and $98 \mathrm{MPa}$ (Hamilton ¿or McKenzie 1965). 


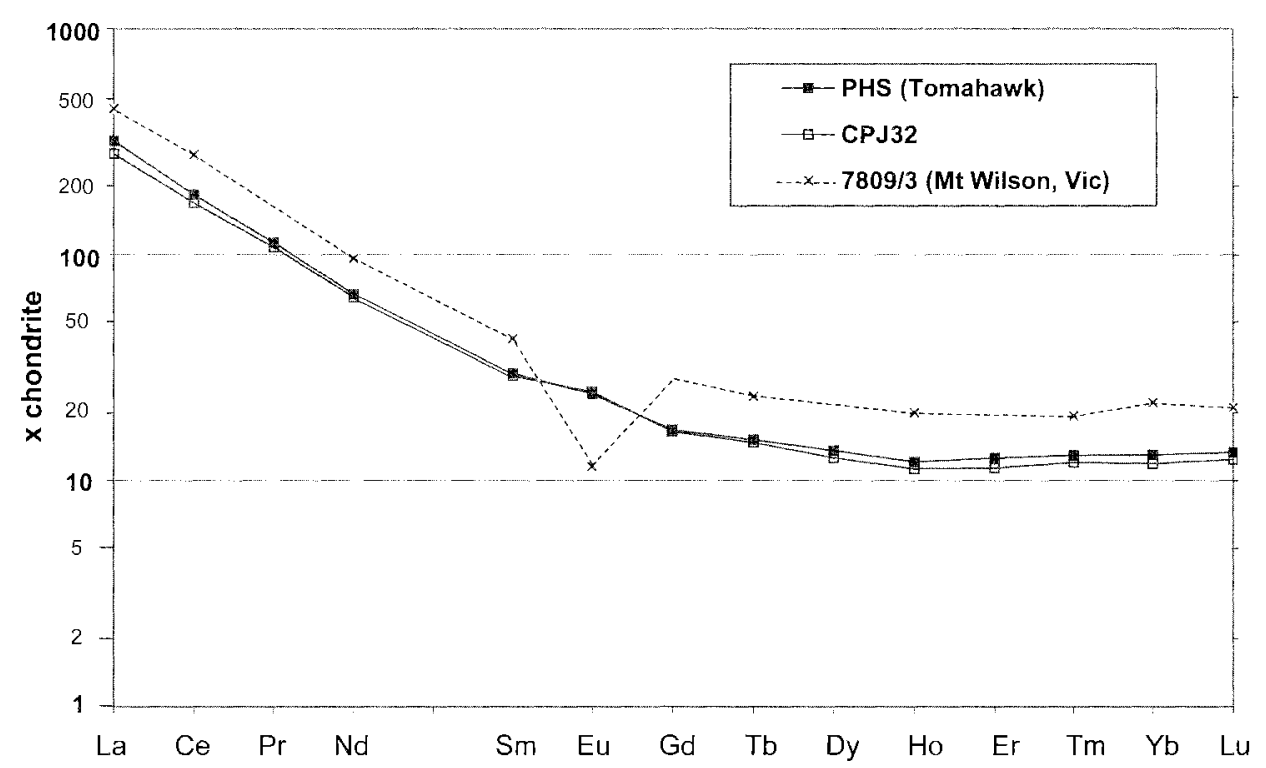

FIG. 12 - Chondrite normalized rare-earth plot for Tomahawk phonolite; data for Mt Wilson phonolite, Victoria (table 9 and Ewart et al. 1985) also plotted (with $G d$ interpolated) for comparison. Chondrite normalization factors after Boynton (1984).

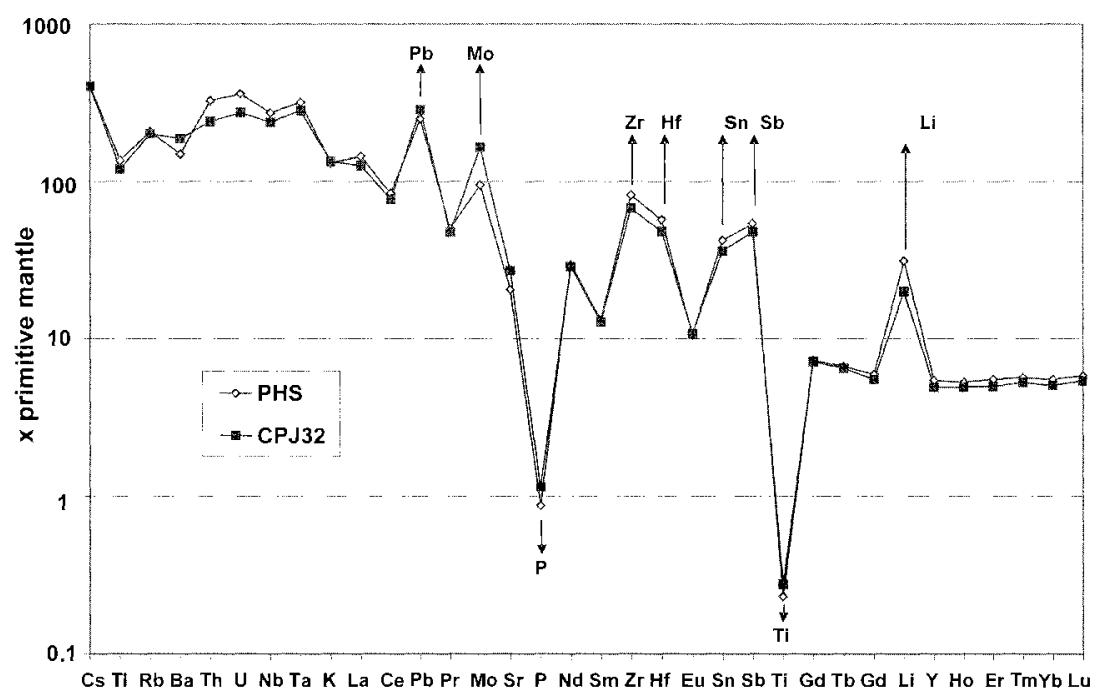

FIG. 13 - Primitive mantle (Sun \& McDonough 1989) normalized trace element spider-diagram for Tomahawk phonolite analyses.

whereas REE (especially MREE and HREE) and Sr have been weakly compatible.

Although the two analyses are very similar, PHS generally has slightly lower levels of compatible trace elements (and also $\mathrm{TiO}_{2}, \mathrm{MgO}$ and $\mathrm{CaO}$ ) and higher levels of incompatible elements, indicating that it is more evolved than CPJ 32.

\section{Petrogenesis}

Although Bailey (1974) maintained that large volumes of phonolitic magma are generated by partial melting in the lower continental crust, most workers ascribe phonolites to the extreme fractionation of silica-undersaturated mafic magmas, such as alkali basalt, basanite or nephelinite. In many studies this is supported by quantitative models of fractional crystallisation and/or direct observation of up- ward changes in chemical composition within a lava pile or tephra sequence (e.g., Garcia et al. 1986, Kyle 1981, Kyle et al. 1992, Le Roex 1990, Price et al. 1985, Thompson et al. 2001, Weaver 1990, Wörner \& Schmincke 1984b). Wilson et al. (1995) and Ablay et al. (1998) suggested crustal assimilation combined with fractional crystallisation (AFC processes) may also occur.

Although lherzolite-bearing olivine nephelinite lavas occur 2-4 km east of the Tomahawk River dyke (Baillie et al. 1978, J. L. Everard, unpublished data; figs 1, 2), they are almost certainly outliers of the Miocene (16 Ma) Ringarooma volcanism (McClenaghan et al. 1982) and are thus unrelated. In the absence of any potentially parental mafic or intermediate rocks, a discussion of the petrogenesis of the Tomahawk River phonolite is speculative. However, some qualitative comments can be made, particularly by comparing its geochemistry with some of the above examples. 
The high MFSE and Rb are generally consistent with strong crystal fractionation, but the lack of $\mathrm{Sr}$ or $\mathrm{Ba}$ depletion or negative Eu anomalies implies that neither alkali feldspar nor plagioclase were major fractionating phases. This suggests an already strongly undersaturated parental basanite or nephelinite with low normative feldspar. The very low levels of compatible elements $(\mathrm{MgO}, \mathrm{Ni}, \mathrm{Cr}$ etc.) suggest dominant olivine, pyroxene and/or amphibole fractionation left a residual felsic liquid that approached the feldspar-nepheline minimum. Strong depletion of P suggests removal of apatite, consistent with apatite inclusions in magnetite and fayalite microphenocrysts.

Fractionation of kaersutitic amphibole from a mafic precursor could account for the concave REE patterns, as MREE are strongly partitioned into amphibole (Sisson 1994), and together with titanomagnetite fractionation may partly account for Ti depletion. Additionally, as noted by Ablay et al. (1978), kaersutite has a low Na:Al ratio and its removal will delay feldspar crystallisation and drive the melt towards peralkaline compositions.

\section{REGIONAL SIGNIFICANCE AND CONCLUSION}

In Tasmania, the Cretaceous was mainly a prolonged period of non-deposition or erosion, and surface environments are rarely preserved. Only localised and relatively minor Cretaceous igneous rocks, mainly intrusions, are known. All are appreciably older than the phonolite, and compositionally dissimilar to it (fig. 10).

Cretaceous appinitic rocks occur in northeast Tasmania at Cape Portland, about $30 \mathrm{~km}$ to the northeast of Tomahawk River, as irregular plug-like intrusions of porphyrite, dykes of lamprophyre and flow remnants of andesite (Jennings \& Sutherland 1969). Sutherland \& Corbett (1974) reported minimum ages ranging from $91 \pm 1 \mathrm{Ma}(\mathrm{K} / \mathrm{Ar})$ to $103 \pm$ $23 \mathrm{Ma}(\mathrm{Rb} / \mathrm{Sr}$ ), and McDougall \& Green (in McClenaghan et al. 1982) obtained $\mathrm{K} / \mathrm{Ar}$ ages of between $102.3 \pm 2.6$ and $101.3 \pm 2.6 \mathrm{Ma}$ from hornblende separates. Small lamprophyre dykes also crop out on Waterhouse Island (Baillie et al. 1978), north of Great Musselroe Bay, Cod Bay and George Rocks (Baillie 1984). Drilling at Cuckoo Creek, behind Great Musselroe Bay, encountered similar dykes, one of which yielded a K/Ar whole rock age of 98.7 $\pm 0.6 \mathrm{Ma}$ (Baillie 1986).

Cretaceous syenites intrude both Late CarboniferousPermian beds and Jurassic dolerite in the Cygnet-Kettering area of southern Tasmania (Ford 1983). Although mainly oversaturated, they include some silica-undersaturated dykes. K/Ar dating of mineral separates indicate an age of $100.5 \pm 0.8 \mathrm{Ma}$ (Evernden \& Richards 1962, McDougall \& Leggo 1965).

The younger ( 8 to $>58 \mathrm{Ma}$ ) Tasmanian Cainozoic basalts contain some relatively evolved nepheline mugearites and mafic nepheline benmoreites (Mg\# 43-53; D. I. 53-58; Sutherland et al. 1989), but none approach the Tomahawk rock (Mg\# 13.2, D. I. 82.8) in this respect.

The Tomahawk River dyke represents a new, albeit very minor, phase of igneous activity for Tasmania. However, some indirect evidence for other Late Cretaceous magmatism exists in the area. This comes from fission-track dating of distinctive pale yellow-brown zircon megacrysts ( 5 grains, $71.3 \pm 5.9 \mathrm{Ma}$ ) found with other younger zircon megacryst suites $(42-53 \pm 2-4 \mathrm{Ma})$ in an alluvial sub-basaltic deposit at Grays Hill, near Branxholm (Australian Museum unpublished data, Geotrack International Report \#169, June 1989). The older zircons lie within error of the Ar-Ar dating of the Tomahawk phonolite.

The 75-81 Ma age of the Tomahawk phonolite overlaps with the main period of the opening of the Tasman Sea, which spans Anomalies 34 to 24 (Gaina et al. 1998), corresponding to about 84 to $52 \mathrm{Ma}$ (Cande \& Kent 1995). Rifting and spreading commenced after $84 \mathrm{Ma}$, along the Tasman margin, off northeastern Tasmania (Duddy \& Green 1992, Sutherland 1994, Royer \& Rollet 1997, O'Sullivan et al. 2000, Norvick \& Smith 2001).

More locally, Cretaceous rifting formed the Boobyalla Sub-basin, a southeasterly extension of the Bass Basin that extends on-shore near the mouth of the Ringarooma River, about $15 \mathrm{~km}$ ENE of the Tomahawk dyke (fig. 1; Moore et al. 1984, Luskin et al. 1989). Geophysical evidence suggests that, in this area, the sub-basin is $600-800 \mathrm{~m}$ thick and bounded by rotational normal faults (Leaman 1973, Leaman $\&$ Symonds 1975). Two drill holes penetrated a non-marine sequence of dolerite-boulder to granule-conglomerate and poorly sorted ferruginous sandstone of mainly Late Cretaceous biostratigraphic age. Angiosperm pollen at the base of the deepest hole $(491 \mathrm{~m})$ indicates an age probably no older than Turonian (89-91 Ma). Thus the Boobyalla Subbasin appears to have been initiated in the Late Cretaceous, considerably later than the remainder of Bass Basin (Luskin et al. 1989) but prior to the opening of the Tasman Sea and the Tomahawk phonolitic magmatism.

Sharples \& Klootwijk (1981) showed that Ordovician Gordon Group Limestone at Ida Bay, in far southern Tasmania, was completely remagnetised in the Late Cretaceous or Early Cainozoic, even though conodont colour indices indicate that the limestone had not been heated beyond $100^{\circ} \mathrm{C}$. This was interpreted as further evidence for a prolonged episode of increased heat flow and widespread remagnetisation in southeastern Australia at this time, possibly related to the rifting prior to opening of Tasman Sea.

Thus, like many other alkaline rocks from continental settings, the Tomahawk River phonolite may be related to rifting and increased heat flow associated with continental break-up.

The Tomahawk alkaline activity does not correlate with any published Late Cretaceous intraplate basaltic activity in eastern Victoria, on the northern side of the intervening Gippsland Basin. Eastern Victorian basaltic intrusions and associated contact metamorphic activity are recorded between 85-95 Ma (McKenzie et al. 1984, Price et al. 1988, Duddy \& Green 1992), but mark an earlier rift-related episode in relation to the initial Tasman spreading period and the Tomahawk magmatism. Phonolitic intrusions appear within some basalt provinces in eastern Victoria, but are not dated and may relate to the older Mesozoic phonolites at Gallows Hill, near Mansfield $(-156 \pm 20 \mathrm{Ma}$, recalculated from McDougall \& Wellman 1976). Proven Late Cretaceous phonolitic magmatism, as at Tomahawk, is rare in eastern Australia. However, a comparative example forms the Mount Ramsay nepheline syenite, near Baralaba, central Queensland (72 Ma; table 11), which may relate to a central volcano plume trace (Sutherland et al. 1996). 


\section{ACKNOWLEDGEMENTS}

The dating work was undertaken as a joint project between the Australian Museum Geodiversity Research Centre and the CSIRO Division of Petroleum Resources (Bentley, Perth, WA). The Ar-Ar data presented in this study were undertaken at the Western Australian Argon Isotope Facility, operated by a consortium of Curtin University and University of Western Australia.

Dr Jo-Anne Wartho (Curtin University) and Andrew Todd (CSIRO Petroleum) are thanked for assistance during K-Ar and ${ }^{40} \mathrm{Ar}-{ }^{39} \mathrm{Ar}$ analyses. R. S. Bottrill, G. R. Green, L. M. Hay, A. J. Hollick, S. Heawood, R. G. Richardson, J. Taheri and R. N. Woolley at Mineral Resources Tasmania are thanked for use of equipment, technical assistance and support. D. Steele and P. Robinson (University of Tasmania) assisted with electron microprobe and performed ICPMS analyses respectively. We also thank Prof. A. J. Crawford and an anonymous reviewer for their advice on the manuscript.

\section{REFERENCES}

Ablay, G.J., Carrol.t, M.R., Palmer, M.R., Marti, J. \& Sparks, R.S.J., 1998: Basanite-phonolite lineages of the TeidePico Viejo Volcanic Complex, Canary Islands. Journal of Petrology 39: 905-936

ANON., 1970: Catalogue of the minerals of Tasmania. Geological Survey Record 9, Tasmania Department of Mines.

BAILEY, D.K., 1974: Otigin of alkaline magmas as a result of anatexis: (b) melting in the deep crust. In Sorensen, H. (Ed.): The Alkaline Rocks. John Wiley \& Sons, London: 436-442.

BALLEY, D.K., 1976: Applications of experiments to alkaline rocks. In Bailey, D. K. \& Macdonald, R. (Eds): The Evolution of Crystalline Rocks. Acadenic Press, London: 419-469.

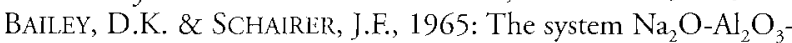
$\mathrm{Fe}_{2} \mathrm{O}_{3}-\mathrm{SiO}_{2}$ at 1 atmosphere, and the petrogenesis of alkaline rocks. Journal of Petrology 7: 114-170.

BAILliE, P.W., 1972: The structure of the granitic rocks of the Little Mt Horror area. Tasmania Department of Mines Technical Reports 15: 32-36.

Bail...IE, P.W., 1984: Geological Atlas 1:50,000 Series. Sheet 85165 (25). Eddystone. Tasmania Department of Mines, Hobart.

BAILIIE, P.W, 1986: Geological Survey Explanatory Report. Sheet 85165 (25). Eddystone. Tasmania Department of Mines, Hobart.

Baillie, P.W., TURner, N.J. \& Cox, S.F., 1978: Geological Atlas 1:50,000 Series. Sheet $8416 S$ (24). Boobyalla. Tasmania Department of Mines, Hobart.

Bonhomme, M.G., Thuizat, R., Pinault, Y., Cl.auer, N., Wendling, R. \& WinkLer, R., 1975: Methode de datation potassium-argon. Appareillage et technique. University of Strasbourg, 53pp.

BOYNTON, W.V., 1984: Geochemistry of the rare earth elements: meteorite studies. In Henderson, P. (Ed.): Rare earth element geochemistry. Elsevier, Amsterdam: 63-114.

CANDE, S.C. \& KENT, D.V., 1995: Revised calibration of the geomagnetic polarity time scale for the Late Cretaceous and Cenozoic. Journal of Geophysical Research 100: 6093 -6095 .

Deer, W.A., Howle, R.A. \& Zussman, J., 1997: Rock-forming minerals. Volume $2 B$, second edition. Double-chain silicates. The Geological Society, London, 764 pp.

DUDDY, I.R. \& GREEN, P.F., 1992: Tectonic development of the Gippsland Basin and environs: identification of key episodes using apatite fission track analysis (AFTA). In Barton, C. (Ed.) Energy, Economics and Environmemt: Gippsland
Basin Symposium, 22-23 June 1992. Australasian Institute of Mining and Metallurgy, Melbourne: 111-120.

EDWARDS, A.B., 1947: Alkali hybrid rocks of Port Cygnet, Tasmania. Proceedings of the Royal Society of Victoria 58: $81-115$.

EVERARD, J.L., 2004: A ground magnetometer survey and compilation of field, petrographic and geochemical data from the Tomahawk River phonolite dyke, northeast Tasmania. Tasmanian Geological Survey Record.

EVERnDEN, J.F. \& RichaRDS, J.R., 1962: Potassium-argon ages in eastern Australia. Journal of the Geological Society of Australia 9: 1-37.

EWart, A., Chappell, B.W. \& Le Maitre, R.W., 1985: Aspects of the mineralogy and geochemistry of the intermediatesilicic Cainozoic volcanic rocks of eastern Australia; part 1: introduction and geochemistry. Australian Journal of Earth Sciences 32: 359-382.

FERGUSSON, A.K., 1978: The crystallization of pyroxenes and amphiboles in some igneous rocks and the presence of a pyroxene compositional gap. Contributions to Mineralogy and Petrology 67: 11-15.

ForD, R.J., 1983: The alkaline rocks of Port Cygnet, Tasmania. Unpublished Ph.D. thesis, University of Tasmania.

Gaina, C., MÜller, D.R., Royer, J.-Y., SToCk, J., Hardebeck, J. \& SYMONDS, P., 1998: The tectonic history of the Tasman Sea: a puzzle with 13 pieces. Journal of Geophysical Research 103: 12413-21433.

Garcia, M.O., Frey, F.A. \& GroOmS, D.G., 1986: Petrology of volcanic rocks from Kaula Island, Hawaii. Contributions to Mineralogy and Petrology 94: 461-471.

GROVES, D.I., 1977: The geology, geochemistry and mineralization of the Blue Tier Batholith. Geological Survey of Tasmania Bulletin 53. Tasmania Department of Mines, Hobart.

HAMILTON, D.L. \& MCKenzIE, W.S., 1965: Phase equilibria studies in the system $\mathrm{NaAlSiO}_{4}$ (nepheline) - $\mathrm{KAISiO}_{4}$ (kalsilite) - $\mathrm{SiO}_{2}$ (quartz). Mineralogical Magazine 34: 214-231.

Heinrichs H. \& Herrmann, A.G., 1990: Praktikum der Analytischen Geochemie. Springer-Verlag, Berlin-Heidelberg, $669 \mathrm{pp}$.

Hess, J.C. \& Lippolt, H.J., 1994: Compilation of K-Ar measurements on HD-B1 standard biotite 1994 status report. Phanerozoic Time Scale. G. S. Odin, Paris. Bulletin of Liaison and Information, IUGS Subcommission of Geochronology 12: 19-23.

JENNINGS, D.J. \& SUTHERI.AND, F.L., 1969: Geology of the Cape Portland area, with special reference to the Mesozoic(?) appinitic rocks. Tasmania Department of Mines Technical Reports 13: 45-81.

JOHNSON, R.W. \& DUGGAN, M.B., 1989: Rock classification and analytical data bases. In Johnson, R.W. (Comp. \& Ed.): Intraplate Volcanism in Eastern Australia and New Zealand. Cambridge University Press, Cambridge: 12-13.

KelLeR, J., 1983: Potassic lavas in the orogenic volcanism of the Mediterranean area. Journal of Volcanology and Geothermal Research 18: 321-335.

KNUTSON, J. (Ed.), 1989: East Australian Volcanic Geology. In Johnson, R.W. (Comp. \& Ed.): Intraplate Volcanism in Eastern Australia and New Zealand. Cambridge University Press, Cambridge: 89-115.

KYLE, P.R., 1981: Mineralogy and petrology of a basanite to phonolite sequence at Hut Point Peninsula, Antarctica, based on core from Dry Valley Drilling Project Drillholes 1,2 and 3. Journal of Petrology 22: $451-500$.

Kyle, P.R., MOORE, J.A. \& ThirLWALl, M.F., 1992: Petrological evolution of anorthoclase phonolite lavas at $\mathrm{Mt}$ Erebus, Ross Island, Antarctica. Joumal of Petrology 33: 849-875.

LEAKE, B.E. (Comp.), 1997: Nomenclature of amphiboles: Report of the Subcommittee on Amphiboles of the International Mineralogical Association, Commission on New Miner- 
als and Mineral Names. American Mineralogist 82: 1019 -1037 .

LEAMAN, D.E., 1973: Summary of geophysical work, Gladstone area. Tasmania Department of Mines Technical Reports 16:89-96.

LEAMAN, D.E. \& SYMONDS, P.A., 1975: Gravity survey of northeastern Tasmania. Geological Survey of Tasmania Paper 2 Tasmania Department of Mines, Hobart.

LE BAS, M.J., LE MAITRE, R.W., STRECKEISEN, A. \& ZANETTIN, B., 1986: A chemical classification of volcanic rocks based on the total alkali silica diagram. Joumal of Petrology 27: $745-750$.

LE MAITRE, R.W., 1976: The chemical variability of some common igneous rocks. Journal of Petrology 17: 589-637.

LE RoEX, A.P., Cliff, R.A. \& ADler, B.J. I., 1990: Tristan da Cunha, South Atlantic: geochemistry and petrogenesis of a basanite-phonolite lava series. Journal of Petrology 31: 779-812.

LIPPARD, S.J., 1973: The petrology of phonolites from the Kenya Rift. Lithos 6: 217-234.

LUDWIG, K.R., 2001: Isoplot/Ex rev. 2.49, a Geochronological Toolkit for Microsoft Excel. Berkeley Geochronology Centre Special Publication 1a.

LUSKIN, A., HOBDAY, D.K. \& BAILLIE, P.W., 1989: Boobyalla Sub-basin. In Burrett, C.F. \& Martin, E.L. (Eds): Geology and Mineral Resources of Tasmania. Geological Society of Australia Inc., Special Publication 15: 356-358.

MCClenaGHAN, M.P. \& WILLLAMS, P.R., 1982: Distribution and characterisation of granitoid intrusions in the Blue Tier area. Geological Survey Paper 4. Tasmania Department of Mines, Hobart.

MCClenaghaN, M.P., TuRner, N.J., BAillie, P.W., Brown, A.V., WILliAMS, P.R. \& MOORE, W.R., 1982: Geology of the Ringarooma- Boobyalla area. Geological Survey of Tasmania Bulletin 61. Tasmania Department of Mines, Hobart.

MCDOUGALL, I. \& HARRISON, T.M., 1999: Geochronology and Thermochronology by the ${ }^{40} A r^{39} A r$ method (2nd Edition). Oxford University Press, New York, 269 pp.

MCDOUGALL, I. \& LEGGO, P.J., 1965: Isotopic age determination on granitic rocks from Tasmania. Joumal of the Geological Society of Australia 12: 295-332.

MCDOUgal, I. \& ROKSANDIC, Z., 1974: Total fusion ${ }^{40} \mathrm{Ar}{ }^{39} \mathrm{Ar}$ ages using HIFAR reactor. Journal of the Geological Society of Australia 21:81-89.

MCDOUGALL, I. \& WELLMAN, P., 1976: Potassium-argon ages for some Australian Mesozoic igneous rocks. Journal of the Geological Society of Australia 23: 1-9.

MCKenziE, D.A., NotT, R.J. \& Bolger, P.F., 1984: Radiometric age determinations. Geological Survey of Victoria Report 74 (unpublished).

MACKENZIE, D.E., BLACK, L.P. \& SUN, S.-S., 1988: Origin of alkali feldspar granites associated with the Poimena granite, northeastern Tasmania. Geochimica et Cosmocbimica Acta 54: 2313-2322.

MARSH, J.S., 1975: Aenigmatite stability in silica- undersaturated rocks. Contributions to Mineralogy and Petrology 50: 135 -144 .

MoOre, W.R., Baille, P.W., Forsyth, S.M., Hudspeth, J.W., Richardson, R.G. \& Turner, N.J., 1984: Boobyalla Sub-basin: a Cretaceous onshore extension of the southern edge of the Bass Basin. APEA Journal 24: 110-1 17.

MORIMOTO, N., 1988: Nomenclature of pyroxenes. American Mineralogist 73: 1123-1133.

NiCKEL, E.H. \& MARK, E., 1965: Arfvedsonite and aegirineaugite from Seal Lake, Labrador. Canadian Mineralogist 8: 185-197.

NOLAN, J., 1966: Melting relations in the system $\mathrm{NaAlSi}_{3} \mathrm{O}_{8}-$ $\mathrm{NaAlSiO}_{4}-\mathrm{NaFeSi}_{2} \mathrm{O}_{6}-\mathrm{CaMgSi}_{2} \mathrm{O}_{6}-\mathrm{H}_{2} \mathrm{O}$ and their bearing on the genesis of alkaline undersaturated rocks. Quarterly Journal of the Geological Society 112: 119-157.

NORvICK, M.S. \& SMITH, M.A., 2001: Mapping the plate tectonic reconstruction of southern and southeastern Australia and implications for petroleum systems. APPEA Journal 41: 15-34.

O'Sullivan, P.B., Mitchell, M.M., O'Sullivan, A.J., KOHN, B.P. \& GLEADOW, A.J.W., 2000: Thermotectonic history of the Bassian Rise, Australia: implications for the breakup of eastern Gondwana along Australia's southeastern margins. Earth and Planetary Science Letters 182: 31-47.

Price, R.C., JOHNSON, R.W., GRAY, C.M. \& FreY, F.A., 1985: Geochemistry of phonolites and trachytes from the summit region of $\mathrm{Mt}$ Kenya. Contributions to Mineralogy and Petrology 89: 394-409.

Price, R.C., Gray, C.M., Nicholls, I.A. \& Day, A., 1988: Cainozoic volcanic rocks. In Douglas, J.G. \& Ferguson, J.A. (Eds): Geology of Victoria. Geological Society of Australia, Victorian Division. Melbourne: 439-451.

QUILTY, P.G. \& WHeller, G.E., 2000: Heard Island and the McDonald Islands: a window into the Kerguelen plateau. Papers and Proceedings of the Royal Society of Tasmania 122(2): 1-12.

ROYER, J-Y. \& ROLLET, N., 1997: Plate-tectonic setting of the Tasmanian region. Australian Journal of Earth Sciences 44: 543-560.

SCHAIRER, J.F., 1950: The alkali feldspar join in the system NaAl$\mathrm{SiO}_{4}-\mathrm{KAlSiO}_{4}-\mathrm{SiO}_{2}$. Journal of Geology 58: 512-517.

SCHAIRER, J.F. \& BOWEN, N.L., 1956: The system $\mathrm{Na}_{2} \mathrm{O}-\mathrm{Al}_{2} \mathrm{O}_{3}$ $\mathrm{SiO}_{2}$. American Journal of Science 254: 129-195.

SHARPLES, C. \& KLOOTWJK, C.T., 1981: Palaeomagnetic results from the Gordon Subgroup of Tasmania: further evidence for a late Cretaceous magnetic overprint in southeastern Australia. Papers and Proceedings of the Royal Society of Tasmania 115: 85-91.

SIMKIN, T. \& SMITH, J.V., 1970: Minor element distribution in olivine. Journal of Geology 78: 304-325.

SISSON, T.W., 1994: Homblende-melt trace-element partitioning measured by ion microprobe. Chemical Geology 117: 331-344.

SOREnSEn, H. (Ed.), 1974: The Alkaline Rocks. John Wiley \& Sons, London. 622 pp.

STEIGER, R.H. \& JÄGER, E., 1977: Subcommission on Geochronology: convention on the use of decay constants in geo- and cosmochronology. Earth and Planetary Science Letters 36: 359-362.

STEPHENSON, P.J., GRIFFIN, T.J. \& SUTHERLAND, F.L., 1980: Cainozoic volcanism in northeastern Australia. In Henderson, R. A. \& Stephenson, P.J. (Eds) The Geology and Geophysics of Northeastern Australia. Geological Society of Australia, Queensland Division: 349-374.

Sun, S.-S. \& MCDONOUGH, W.F., 1989: Chemical and isotopic systematics of oceanic basalts: implications for mantle composition and processes. In Saunders, A. D. \& Norry, M. J. (Eds) Magmatism in the Ocean Basins. Geological Society of London Special Publication 42: 313-346.

SUTHERLAND, F.L., 1976: Cainozoic volcanic rocks. In Leaman, D.E. Geological Survey Explanatory Report. Sheet 82 (8312S). Hobart. Tasmania Department of Mines, Hobarr: $35-36$.

SUTHERLAND, F.L., 1994: Tasman Sea evolution and hotspot trails. In Van der Lingen, G.J., Swanson, K.M. \& Muir, R.J. (Eds): Evolution of the Tasman Sea Basin. A.A. Balkema, Rotterdam: 35-51.

Sutherland, F.L. \& CORBETT, E.B., 1974: The extent of Upper Mesozoic igneous activity in relation to lamprophyric intrusions in Tasmania. Papers and Proceedings of the Royal Society of Tasmania 107: 175-190.

SuTherland, F.L., EwarT, A., RaYNor, L.R., Hollis, J.D. \& MCDONOUGH, W.D., 1989: Tertiary basaltic magmas and the Tasmanian lithosphere. In Burrett, C.F. \& Martin, E.L. (Eds): Geology and Mineral Resources of Tasmania. Geological Society of Australia Special Publication 15: 386-398. 
Sutherland, F.L., Robertson, A.D., Barron, B.J. \& PogSON, R.E., 1996: The Rockhampton plume and irs late Mesozoic trace? In Mesozoic Geology of the Eastern Australian Plate, Mesozoic 96 Conference, 23 - 26 September, Brisbane. Geological Society of Australia Extended Abstracts 43: 519-527.

TAHeri, J. \& BOTTRILL, R., 1999: Porphyry and sedimentaryhosted gold deposits near Cygnet: new styles of gold mineralisation in Tasmania. Tasmanian Geological Survey Record 1999/01.

THOMPSON, G.M., SMITH, 1.E.M. \& MALPAS, J.G., 2001: Origin of oceanic phonolites by crystal fractionation and the problem of the Daly gap: an example from Rarotonga. Contributions to Mineralogy and Petrology 142: 336-346.

WEAVER, B.L., 1990: Geochemistry of highly under-saturated oceanic island basalt suites from the South Atlantic Ocean: Fernando de Noronha and Trinidade islands. Contributions to Mineralogy and Petrology 105: 502-515.

WiLsON, M., DOWNes, H. \& CEBRIA, J., 1995: Contrasting fractionation trends in coexisting continental alkaline magma series; Cantal, Massif Central, France. Journal of Petrology 36: 1723-1753.
WONES, D.R. \& EUGSTER, H.P., 1965: Stability of biotite: experiment, theory and application. American Mineralogist 50: $1228-1272$.

WÖRNER, G. \& SCHMINCKE, H.-U., 1984a: Mineralogical and chemical zonation of the Laacher See tephra sequence (East Eifel, W. Germany). Journal of Petrology 25: 805-835.

WÖnner, G. \& SCHMINCKE, H.-U., 1984b: Petrogenesis of the zoned Laacher See tephra. Journal of Petrology 25: $836-851$.

Young, G.C. \& Laurie, J.R., (Eds), 1996: An Australian Phanerozoic Timescale. Oxford University Press, Melbourne, $279 \mathrm{pp}$.

YU Z., ROBINSON P. \& MCGOLDRICK P., 2001: An evaluation of methods for the chemical decomposition of geological materials for trace element determination using ICP-MS. Geostandards Newsletter, the Journal of Geostandards and Geoanalysis 25: 199-217.

(accepted 2 February 2004) 